\title{
Gross-Neveu Model Through Convergent Perturbation Expansions
}

\author{
K. Gawędzki ${ }^{1,2}$ \\ C.N.R.S., I.H.E.S., F-91440 Bures-sur-Yvette, France
}

A. Kupiainen ${ }^{2}$

Research Institute for Theoretical Physics, University of Helsinki, Helsinki, Finland

\begin{abstract}
We construct a continuum limit for the effective low energy Lagrangians of the Gross-Neveu model in two euclidean dimensions by showing that they are related to each other through convergent perturbation expansions. This provides a rigorous control of the ultraviolet problem in a renormalizable quantum field theory.
\end{abstract}

\section{Introduction}

It has been observed, back in the fifties, that in quantum field theories with fermions, the perturbation expansion is better behaved than in purely bosonic ones. Indeed, it was noted [1] that for QED with finite ultraviolet (UV) and infrared (IR) cutoffs the expansion in fact converges, in sharp contrast with bosonic theories where the perturbation series is only asymptotic. However, the situation is much less clear in renormalizable theories of fermions once the UV cutoff has been removed and the renormalization subtractions performed. The nice property of the cutoff theory seems to be lost as is indicated by the conjectured presence of renormalon singularities in the Borel transform of the renormalized perturbation series of any asymptotically free model, regardless of statistics [2]. In the present paper, we show that important traces of the convergence for cutoff fermions remain when the cutoff is removed. In fact, we construct a local renormalizable asymptotically free theory of fermions by a successive application of convergent perturbation expansions, one per each limited range of momentum fluctuations.

We consider the Gross-Neveu models $[3,4]$ in two (euclidean) dimensions: the perturbatively renormalizable, asymptotically free field theories of Dirac fermions $\psi_{\alpha}^{i}(x), \alpha=0,1, i=1, \ldots, N$, with $N>1$ internal [U(N) "flavor"] symmetry indices, and the action given by

$$
S=\int d x\left[\bar{\psi} i \not \partial \psi-g(\bar{\psi} \psi)^{2}\right],
$$

1 Supported in part by the National Science Foundation under Grant MCS-81-20833

2 Supported in part by the National Science Foundation under Grant PHY-82-03669 
or variations thereof. It is easy to show that such theories, say on a lattice and in a finite box, have a convergent expansion in powers of $g$. In order to understand this, first consider a free massive charged bosonic theory on the lattice. The Green function

$$
\left\langle\prod_{i=1}^{n} \phi\left(x_{i}\right) \phi^{*}\left(y_{i}\right)\right\rangle=\sum_{\pi \in S_{n}} \prod_{i=1}^{n} G\left(x_{i}-y_{\pi(i)}\right),
$$

is a sum of $n$ ! terms ( $G$ is the propagator and $S_{n}$ the permutation group of $n$ elements) and e.g. for $x_{i}=y_{i} \equiv x$ will increase like $O(n !)$ with $n$. This leads to the well known $O(n$ !) behavior of the perturbative expansion of the Green functions in the $g\left(\phi^{*} \phi\right)^{2}$ model, for the $n^{\text {th }}$ order in $g$. For lattice fermions, the situation is drastically different. Now (ignore indices)

$$
\left\langle\prod_{i=1}^{n} \psi\left(x_{i}\right) \bar{\psi}\left(y_{i}\right)\right\rangle=\sum_{\pi \in S_{n}}(-1)^{\pi} \prod_{i=1}^{n} G\left(x_{i}-y_{\pi(i)}\right)=\operatorname{det} G\left(x_{i}-y_{j}\right),
$$

which is bounded, e.g. with the use of the Hadamard inequality, by $O(1)^{n}$ if $G$ has mass or is confined to a finite volume. This observation leads to the convergence of the perturbation expansion for our model with cutoffs. What happened? The point

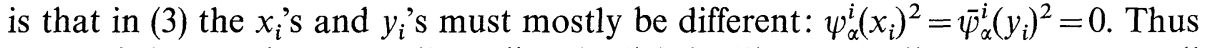
most of the graphs [even discarding $\left.(-1)^{\pi}\right]$ in (3) necessarily carry very small factors due to the falloff of $G$ (or in finite volume eventually, for $n$ big enough, vanish). Indeed. The divergence of the perturbation series for bosons is due to the fact that arbitrary high powers of the field at the same point may occur. For fermions, exclusion principle prevents that.

How does this observation survive the process of renormalization? Following the Wilson renormalization group (RG) approach [5], we shall carry out the functional integral with UV cutoff $\Lambda$ as a sequence of fixed momentum-scale integrals by slicing the interval $\left[0, \Lambda=L^{n_{0}} \mu\right]$ into intervals $\left[L^{n-1} \mu, L^{n} \mu\right][\mu$ is a fixed scale, $L=O(1)]$, and integrating out successively fluctuations on these scales (from $n=n_{0}$ to $n=1$ ). Thus we produce a sequence of effective actions

$$
S^{\Lambda} \rightarrow S_{L^{-1} \Lambda}^{\Lambda \mathrm{EFF}} \rightarrow \ldots \rightarrow S_{L \mu}^{\Lambda \mathrm{EFF}} \rightarrow S_{\mu}^{\Lambda \mathrm{EFF}},
$$

$S_{\tilde{\Lambda}}^{\Lambda \mathrm{EFF}}$ describing the theory with action $S^{\Lambda}$ at momenta $\leqq \tilde{\Lambda}$ (vaguely). The crucial observation is that each such step is a finite (in dimensionless units) UV and IR cutoff integration and may be performed perturbatively through a convergent expansion. Now existence of the continuum limit means that $S^{\Lambda}$ may be chosen appropriately as a function of $\Lambda$ so that for $\tilde{\Lambda}$ arbitrary but fixed,

$$
S_{\tilde{\Lambda}}^{\Lambda \mathrm{EFF}} \underset{\Lambda \rightarrow \infty}{\longrightarrow} S_{\tilde{\Lambda}}^{\infty} \mathrm{EFF},
$$

which is a well defined action describing the continuum local field theory at momenta $|p| \leqq \tilde{\Lambda}$. In the present paper, we show that for the Gross-Neveu models, the limit (5) exists as a convergent power series in $\psi, \bar{\psi}$ both in finite and in infinite volume:

$$
S_{\tilde{\Lambda}}^{\mathrm{EFF}}=\sum_{m=0}^{\infty} \int d x_{1} \ldots d x_{m} d y_{1} \ldots d y_{m} S_{\tilde{\Lambda}}^{m}(\mathbf{x}, \mathbf{y}) \prod_{i} \bar{\psi}\left(x_{i}\right) \psi\left(y_{i}\right)
$$

(what we mean by convergence here is explained in Sect. 4). 
The main idea of our RG analysis is similar to Wilson's analysis [6] of a toy fermionic model where boundedness of fermions was also heavily used. The coefficients $S_{\tilde{\Lambda}}^{m}$ in (6) are functions of the renormalized coupling $g \geqq 0$ of the model (which we take small) and may be expanded in powers of $g$. These renormalized perturbation series should not converge. We hope to exhibit the presence of renormalon singularities in their Borel transforms using our non-perturbative analysis [7]. The instanton singularities present in bosonic theories are absent here, reflecting again boundedness of fermions.

The rigorous $\mathrm{RG}$ analysis of the effective actions can be easily extended to the Green functions. For models (1) with mass term added, one can obtain $\Lambda=\infty$ infinite volume Green functions and control their short distance (scaling with logarithmic corrections) and (massive) long distance behaviour. This will provide first models of renormalizable asymptotically free theories satisfying all euclidean and (after analytic continuation) Wightman axioms. For the massless models, it is easy to construct $\Lambda=\infty$ finite volume Green functions. In the infinite volume, one expects dynamic mass generation in this case, at least for large number $N$ of flavors $[4,8]$. The Green functions will be analyzed in a separate paper.

Finally, one should mention that a lot is known about the chirally invariant version of the Gross-Neveu model discussed below: by the Bethe ansatz methods its spectrum has been obtained [9] and its $S$-matrix has been found [10] (but not the Green functions). The present paper provides quite a simple picture of what renormalization is all about in such asymptotically free models. Certainly, it is a simpler one than the standard perturbative approach and moreover rigorous and non-perturbative. It also seems that the methods developed here may be carried on to large $N$, the number of flavors, uniformly in $N$, allowing rigorous control of the mass generation in the massless models and the construction of the nonrenormalizable three-dimensional theory for $N$ large but finite. Also by similar methods, only with more sophisticated cluster expansions replacing the simpleminded perturbative ones which diverge for bosons, one is able to control the continuum limit of $-\lambda \phi_{4}^{4}$ euclidean theory [11].

When the paper was finished, we learned that J. Feldman, J. Magnen, V. Rivasseau, and R. Sénéor announced similar results [17].

\section{The Models}

We take the bare euclidean action with momentum cutoff $\Lambda$ to be

$$
S^{\Lambda}=S^{O \Lambda}+S^{I \Lambda}
$$

with the free part

$$
S^{O \Lambda}=\int d x \bar{\psi} i \phi_{\Lambda} \psi,
$$

and the interaction for the simplest model

$$
\begin{aligned}
S^{I \Lambda}= & z^{\Lambda} \int d x \bar{\psi} i \not \partial \psi+m^{\Lambda} \int d x \bar{\psi} \psi \\
& -g^{\Lambda} \int d x(\bar{\psi} \psi)^{2} .
\end{aligned}
$$


Here $\psi=\left(\psi_{\alpha}^{i}\right), \not \partial=\partial_{\mu} \gamma^{\mu}$ with anti-hermitian $2 \times 2$ Dirac matrices $\gamma^{\mu}$ satisfying $\left\{\gamma^{\mu}, \gamma^{\nu}\right\}=-2 \delta^{\mu \nu}$, and

$$
\left(i \nexists_{\Lambda}\right)^{-1}\left(x_{1}-x_{2}\right)=(2 \pi)^{-2} \int d p e^{i p\left(x_{1}-x_{2}\right)} \frac{\not p}{p^{2}} e^{-p^{2} / \Lambda^{2}} \equiv \mathscr{G}_{\Lambda}\left(x_{1}-x_{2}\right),
$$

is the free momentum cutoff propagator. When $m^{\Lambda}=0$, the action $S^{\Lambda}$ has the discrete chiral invariance

$$
\psi \rightarrow i \gamma^{5} \psi, \quad \bar{\psi} \rightarrow i \bar{\psi} \gamma^{5},
$$

where $\gamma^{5}=i \gamma^{0} \gamma^{1}$, which forbids mass terms to appear in any of the effective actions. We shall also consider the "chirally invariant" version of the model, where the quartic interaction term in (9) is replaced by

$$
-g^{\Lambda} \int d x\left[(\bar{\psi} \psi)^{2}-\left(\bar{\psi} \gamma^{5} \psi\right)^{2}\right]-g_{v}^{\Lambda} \int d x\left(\bar{\psi} \gamma^{\mu} \psi\right)^{2}
$$

If $m^{\Lambda}=0$, this model has the continuous chiral symmetry

$$
\psi \rightarrow e^{i \theta \gamma_{5}} \psi, \quad \bar{\psi} \rightarrow \bar{\psi} e^{i \theta \gamma_{5}}
$$

$\left((11)\right.$ is a special case of $(13)$ for $\left.\theta=\frac{\Pi}{2}\right)$.

In order to have finite functional integrals to start with, we shall make a replacement

$$
\psi(x)=(2 \pi)^{-2} \int d p e^{i p x} \hat{\psi}(p) \rightarrow \sum_{p} \ell^{-2} e^{i p x} \hat{\psi}(p)
$$

where $p \in \frac{2 \pi}{\ell} \mathbb{Z}^{2}, p \neq 0$ and $|p|<\ell \Lambda^{2}$ (and the same for $\bar{\psi}$ ). Thus we set the theory on the finite periodic box of side $\ell$ and throw out the zero momentum and the large momentum modes, retaining only finite number of degrees of freedom [for $\ell$ large, the important cutoff is still $\Lambda$ of $(10)] . \hat{\psi}_{\alpha}^{i}(p)$ and $\hat{\bar{\psi}}_{\alpha}^{i}$ generate a finite dimensional Grassmann algebra. Later, it will be convenient to use a generic notation for $\psi$ and $\bar{\psi}$ : we view the components of $\psi$ and $\bar{\psi}$ as components of a single field denoted by $\tilde{\psi} . \tilde{\psi}$ thus carries Lorentz, charge and flavor indices, collectively denoted by the subscript in $\tilde{\psi}_{a}$ and usually suppressed. We shall widely use a shorthand notation, so e.g. $\tilde{\psi}(\mathbf{x}, \mathbf{a})$ or $\tilde{\psi}(\mathbf{x})$ or $\tilde{\psi}(x)^{m}$ will stand for $\tilde{\psi}_{a_{1}}\left(x_{1}\right) \ldots \tilde{\psi}_{a_{m}}\left(x_{m}\right)$, the latter if $x_{i}=x$. With the additional cutoff (14), the numerator and the denominator in the cutoff Green functions as given by the Berezin integral

$$
\langle\tilde{\psi}(\mathbf{x})\rangle=\int D \tilde{\psi} \tilde{\psi}(\mathbf{x}) e^{-S^{\Lambda}(\tilde{\psi})} / \int D \tilde{\psi} e^{-S^{\Lambda}(\tilde{\psi})},
$$

are finite $\left(D \tilde{\psi}=\prod_{a, p} d \tilde{\psi}_{a}(p)\right)$. As our later analysis will show, the denominator is non-zero so that $(15)$ is well defined.

\section{Effective Actions}

The analysis of the UV $(\Lambda \rightarrow \infty)$ (and infinite volume) limit of $(15)$ is based on lowering the cutoff through the process of integrating out high momentum fields. We write

$$
\mathscr{G}_{\Lambda}(x-y)=\mathscr{G}_{\tilde{\Lambda}}(x-y)+\mathscr{T}(x-y)
$$


where $\mathscr{T}$ has the cutoff function $\left(e^{-p^{2} / \Lambda^{2}}-e^{-p^{2} / \tilde{\Lambda}^{2}}\right)$. Actually, with the full cutoff (14), $\mathscr{G}_{\tilde{\Lambda}}$ in (16) still has $|p|<\ell \Lambda^{2}$. Our estimates will be uniform in $\ell$ and from now on, we shall work in the infinite volume notation if no confusion arises. Equation (16) allows us to split the fields $\psi$ and $\bar{\psi}$ into high and low frequency parts,

$$
\tilde{\psi}(x)=\tilde{\psi}^{\prime}(x)+\tilde{\zeta}(x),
$$

so that for any functional $F$ on the (finite) Grassmann algebra,

$$
\int d \mu_{\mathscr{G}_{A}}(\tilde{\psi}) F(\tilde{\psi})=\int d \mu_{\mathscr{G}_{\tilde{\Lambda}}}\left(\tilde{\psi}^{\prime}\right) \int d \mu_{\mathscr{T}}(\tilde{\zeta}) F\left(\tilde{\psi}^{\prime}+\tilde{\zeta}\right)
$$

where we use the notation $d \mu_{\mathscr{G}}(\tilde{\psi})$ for the "gaussian measure"

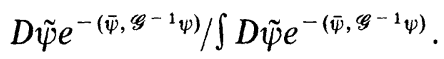

Now the effective action for momenta $\leqq \tilde{\Lambda}, S_{\tilde{\Lambda}}^{\Lambda \mathrm{EFF}}(\tilde{\psi})$, is defined by

$$
S_{\tilde{\Lambda}}^{\Lambda \mathrm{EFF}}\left(\tilde{\psi}^{\prime}\right)=\int d x \bar{\psi}^{\prime} i \not_{\tilde{\Lambda}} \psi^{\prime}-\log \int d \mu_{\mathscr{T}}(\tilde{\zeta}) e^{-S^{I \Lambda}\left(\tilde{\psi}^{\prime}+\tilde{\zeta}\right)}+\text { const . }
$$

(We shall see that the logarithm is well defined, note that $\exp \left[-S_{\tilde{\Lambda}}^{\Lambda \mathrm{EFF}}\right]$ is.) $S_{\tilde{\Lambda}}^{\Lambda \mathrm{EFF}}$ has cutoff $\tilde{\Lambda}$ and residual dependence on $\Lambda$. It will be in general non-local with couplings involving arbitrarily many $\tilde{\psi}$ 's.

Let us state our main results. Consider the model (9) with $m^{\Lambda}=0$. For small $g \geqq 0$ take the bare couplings as

$$
\left(g^{\Lambda}\right)^{-1}=g^{-1}-\beta_{2} \log \frac{\Lambda}{\mu}+\frac{\beta_{3}}{\beta_{2}} \log \left(1-g \beta_{2} \log \frac{\Lambda}{\mu}\right),
$$

where $\mu$ is a fixed scale and

with

$$
z^{\Lambda}=-\frac{\gamma_{2}}{\beta_{2}} g^{\Lambda}
$$

$$
\beta_{2}=-2(N-1) \pi^{-1}, \quad \gamma_{2}=(2 N-1)\left(2 \pi^{2}\right)^{-1},
$$

and $\beta_{3}$ being a computable coefficient $\left(\beta_{2}, \beta_{3}\right.$, and $\gamma_{2}$ are first coefficients of the RG $\beta$ and $\gamma$ functions).

Theorem. The effective actions $S_{\tilde{\Lambda}}^{\mathrm{EFF}}$ on scales $\tilde{\Lambda} \geqq \mu$ may be written as

$$
\begin{aligned}
S_{\tilde{\Lambda}}^{\Lambda \mathrm{EFF}}(\tilde{\psi})= & \int d x\left(\bar{\psi} i \phi_{\tilde{\Lambda}} \psi+z_{\tilde{\Lambda}}^{\Lambda} \bar{\psi} i \not \partial \psi-g_{\tilde{\Lambda}}^{\Lambda}(\bar{\psi} \psi)^{2}\right) \\
& +\sum_{\mathbf{m}, \mathbf{r}} \int d \mathbf{x} S_{\tilde{\Lambda}}^{\Lambda \mathbf{m r}}(\mathbf{x}) \prod_{i=1}^{q}\left(\partial \tilde{\psi}\left(x_{i}\right)\right)^{r_{i}} \tilde{\psi}\left(x_{i}\right)^{m_{i}-r_{i}},
\end{aligned}
$$

where $\mathbf{m}=\left(m_{i}\right)_{i=1}^{q}, 0<m_{i}<4, \mathbf{r}=\left(r_{i}\right)_{i=1}^{q}, r_{i}=0$ or 1 . Only even $m \equiv \sum m_{i}$ occur. For $m=2, r \equiv \sum r_{i}=2$ and for $m=4, r \geqq 1$. The running coupling constant $g_{\tilde{\Lambda}}^{4}$, the running wave function renormalization $1+z_{\Lambda}^{A}$ and the kernels $S_{\Lambda}^{\Lambda m r}$ have continuum $(\Lambda \rightarrow \infty)$ and infinite volume limits

$$
\begin{aligned}
g_{\tilde{\Lambda}}^{\infty} & =g^{\tilde{\Lambda}}+o\left(\left(g^{\tilde{\Lambda}}\right)^{2}\right), \\
z_{\tilde{\Lambda}}^{\infty} & =-\frac{\gamma_{2}}{\beta_{2}} g^{\tilde{\Lambda}}+O\left(\left(g^{\tilde{\Lambda}}\right)^{2}\right), \\
S_{\tilde{\Lambda}}^{\infty \mathbf{m r}}(\mathbf{x}) & =\left(\frac{\tilde{\Lambda}}{\mu}\right)^{2 q-\frac{m}{2}-r} H_{\tilde{\Lambda}}^{\infty \mathbf{m r}}\left(\frac{\tilde{\Lambda}}{\mu} \mathbf{x}\right),
\end{aligned}
$$


where

$$
\left\|H_{\tilde{\Lambda}}^{\infty \mathbf{m r}}\right\| \equiv \int d x_{2} \ldots d x_{m}\left|H_{\tilde{\Lambda}}^{\infty \mathbf{m r}}(\mathbf{x})\right| e^{\mu \mathscr{L}(\mathbf{x})} \leqq \begin{cases}C\left(g^{\tilde{\Lambda}}\right)^{2}, & m<8 \\ C^{m}\left(g^{\tilde{A}}\right)^{3+\varepsilon(m-8)}, & m \geqq 8\end{cases}
$$

with $\mathscr{L}(\mathbf{x})$ being the length of the shortest tree on the points of $\mathbf{x}$ and possibly other points.

Remarks. 1. Component and derivative indices are suppressed and implicitly summed over above. $S_{\tilde{\Lambda}}^{\Lambda \mathrm{mr}}$ have all the (anti-)symmetries of the product of $\tilde{\psi}$ 's in (23).

2. $H_{\tilde{\Lambda}}^{\mathrm{mr}}$ are $S_{\tilde{\Lambda}}^{\mathrm{mr}}$ written in dimensionless variables for easier comparison at different values of $\tilde{\Lambda}$.

3. Equation (21) describes the behavior of the bare coupling constant in the leading and subleading logarithms approximation obtained by solving the RG equations with $\beta$ function given to $O\left(g^{3}\right)$. We could take $z^{A}$ equal to zero, but (22) has a more convenient form. Note how these exact expressions suffice for renormalization.

4. To the order $O\left(g^{\tilde{A}}\right), S_{\tilde{\Lambda}}^{\mathrm{EFF}}$ is equal to the bare action $S^{\tilde{\Lambda}}$ with cutoff $\tilde{\Lambda}$, the difference being a smąll approximately local perturbation. The bare and renormalized trajectories almost coincide.

5. The $S_{\tilde{\Lambda}}^{\mathrm{mr}}$ terms have positive dimension (are irrelevant under the RG). In particular, the local part of the $m=2$ term in (23) starts with $\int(\bar{\psi} \phi \psi)^{2}$ and that of the $m=4$ one with $(\bar{\psi} \not \psi)^{2}$. Thus no negative dimension or dimensionless interactions are generated.

6. For the chirally invariant model, we take $g^{\Lambda}$ and $z^{\Lambda}$ as in (21) and (22), with $\beta_{2}=-\frac{2 N}{\pi}, \gamma_{2}=\frac{2}{\pi^{2}}\left(N-\frac{1}{N}\right), \beta_{3}$ computable and $g_{v}^{\Lambda}=-\frac{1}{N} g^{\Lambda}$. Now the local quartic term in (23) becomes

$$
-g_{\tilde{\Lambda}}^{\Lambda} \int d x\left[(\bar{\psi} \psi)^{2}-\left(\bar{\psi} \gamma_{5} \gamma\right)^{2}\right]-g_{v \tilde{\Lambda}}^{\Lambda} \int d x\left(\bar{\psi} \gamma^{\mu} \psi\right)^{2},
$$

and when $\Lambda \rightarrow \infty, g_{v \tilde{\Lambda}}^{\Lambda} \rightarrow g_{v \tilde{\Lambda}}^{\infty}=-\frac{1}{N} g^{\tilde{\Lambda}}+O\left(\left(g^{\tilde{\Lambda}}\right)^{2}\right)$. Again, we could also take $z^{\Lambda}=0=g_{v}^{\Lambda}$.

7. In the case of the massive model, we take for $m$ posituve

$$
m^{\Lambda}=m \cdot\left(g^{\Lambda} / g\right)^{-v}
$$

with $v=\frac{N-1 / 2}{N-1}$ for model (9) and $v=1-\frac{1}{N^{2}}$ for model (12). It is then possible not only to control the continuum limit of the effective actions but also to construct, for $0<g<g_{0}(m / \mu)$, the infinite volume continuum Green functions again by applying inductive convergent expansions. Note again that an exact formula for the bare mass may be given.

\section{The Renormalization Group Transformation}

It is convenient to use dimensionless variables by introducing "Hamiltonians" $H_{\tilde{\Lambda}}^{\Lambda} \mathrm{EFF}$,

$$
S_{\tilde{\Lambda}}^{\Lambda \mathrm{EFF}}(\tilde{\psi})=H_{\tilde{\Lambda}}^{\Lambda \mathrm{EFF}}\left(\left(\frac{\mu}{\tilde{\Lambda}}\right)^{1 / 2} \tilde{\psi}\left(\frac{\mu}{\tilde{\Lambda}} \cdot\right)\right)
$$


The initial Hamiltonian is

$$
\begin{aligned}
H^{\Lambda}(\tilde{\psi}) & =\int d x\left[\bar{\psi} i \phi_{\mu} \psi+z^{\Lambda} \bar{\psi} i \not \psi \psi+m^{\Lambda} \frac{\mu}{\Lambda} \bar{\psi} \psi-g^{\Lambda}(\bar{\psi} \psi)^{2}\right] \\
& \equiv H^{0}(\tilde{\psi})+H^{I \Lambda}(\tilde{\psi})
\end{aligned}
$$

and $[$ see $(20)]$

$$
\begin{aligned}
& H_{\tilde{\Lambda}}^{\Lambda \mathrm{EFF}}(\tilde{\psi}) \equiv\left(R_{\Lambda / \tilde{\Lambda}} H^{\Lambda}\right)(\tilde{\psi})=H^{0}(\tilde{\psi})
\end{aligned}
$$

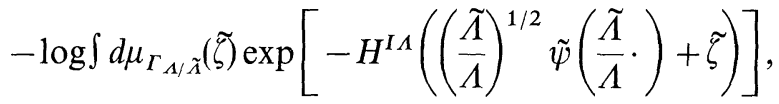

where in momentum space

$$
\hat{\Gamma}_{\Lambda / \tilde{\Lambda}}(p)=\frac{\not p}{p^{2}}\left(\exp \left[-p^{2} / \mu^{2}\right]-\exp \left[-p^{2} \Lambda^{2} / \mu^{2} \tilde{\Lambda}^{2}\right]\right) .
$$

Below, we shall put $\mu=1$ for the sake of simplicity. In order to prove (23), we take $\Lambda=L^{n_{0}}, \tilde{\Lambda}=L^{n}$ and compute $H_{\tilde{\Lambda}}^{\Lambda^{\mathrm{EFF}}} \equiv H_{n}^{n_{0} \mathrm{EFF}}$ in $n_{0}-n$ inductive steps using the semigroup property of the RG transformation,

$$
R_{\Lambda / \tilde{\Lambda}}=\left(R_{L}\right)^{n_{0}-n} .
$$

Thus we are led to study in general the transformation $T_{L} \equiv T\left(\Gamma \equiv \Gamma_{L}\right)$

$$
\left(T H^{I}\right)(\tilde{\psi})=-\log \int d \mu_{\Gamma}(\tilde{\zeta}) \exp \left[-H^{I}\left(L^{-1 / 2} \tilde{\psi}\left(L^{-1} \cdot\right)+\tilde{\zeta}\right)\right] .
$$

It is instructive to study the first application of $T$ on $H^{I \Lambda} \equiv H^{I n_{0}}$ (with $m^{\Lambda}=0$ ). Recall that $(\Lambda, \ell<\infty)$ the functional integral in (33) is a well defined element of a finite-dimensional Grassmann algebra. As we shall see later, the $c$-number part of it is positive and hence the logarithm is also well defined. More interestingly, we may express it as a finite perturbation series,

$$
\begin{aligned}
\left(T H^{I n_{0}}\right)\left(\tilde{\psi}^{\prime}\right) \equiv & H_{n_{0}-1}^{I n_{0}}\left(\tilde{\psi}^{\prime}\right)=-\sum_{k, \ell}(k ! \ell !)^{-1}\left(-z^{n_{0}}\right)^{k}\left(g^{n_{0}}\right)^{\ell} \\
& \cdot \int d x_{1} \ldots d x_{k+\ell}\left\langle\prod_{1}^{k}(\bar{\psi} i \not \phi \psi)\left(x_{i}\right) \prod_{k+\ell}^{\ell}(\bar{\psi} \psi)^{2}\left(x_{i}\right)\right\rangle_{\Gamma}^{T} .
\end{aligned}
$$

Here $\tilde{\psi}(x)=L^{-1 / 2} \tilde{\psi}^{\prime}\left(L^{-1} x\right)+\tilde{\zeta}(x)$ and \langle\rangle$_{\Gamma}^{T}$ is the truncated expectation in the measure $d \mu_{\Gamma}(\tilde{\zeta})$. Let us express (34) as a series in $\tilde{\psi}^{\prime}$ [recall notation of (23)]:

$$
H_{n_{0}-1}^{I n_{0}}\left(\tilde{\psi}^{\prime}\right)=\sum_{\mathbf{m r}} \int d \mathbf{x} H^{\mathbf{m r}}(\mathbf{x}) \prod_{1}^{q}\left(\partial \tilde{\psi}^{\prime}\left(x_{i}\right)\right)^{r_{i}}\left(\tilde{\psi}^{\prime}\left(x_{i}\right)\right)^{m_{t}-r_{i}} .
$$

$H^{\mathbf{m r}}(\mathbf{x})$ are given as sums of Feynman graphs with $m$ external lines at $q$ points $x_{i}, z^{\Lambda}$ and $g^{\Lambda}$ vertices of (29) and propagators $\Gamma$ on the internal lines. As discussed in the introduction, such expansions converge due to the exclusion principle and exponential decay of $\Gamma$,

$$
|\Gamma(x-y)| \leqq C(a, L) e^{-a|x-y|},
$$

uniformly in cutoff $\ell$, as is readily seen from its Fourier transform, see (31). The estimates of truncated $\tilde{\zeta}$ expectations which provide a technical basis for the convergence proof given in Sect. 6 and which exhibit a striking contrast between the fermionic and the bosonic cases are discussed in the appendices. 


\section{The $\beta$ and $\gamma$ Functions}

In this section explicit bounds for $S_{\tilde{\Lambda}}^{\mathrm{EFF}}$ are found assuming suitable convergence of the perturbation series, which is established in Sect. 6. We start quite explicitly with the lowest orders since these are crucial for the RG flow and illustrate general analysis.

A. Extracting Local Couplings: $2^{\text {nd }}$ Order. The term linear in $H^{I^{n_{0}}}$ is [suppress superscript $n_{0}$ : consider the first step of iteration (34)]

$$
z \int d x \bar{\psi}^{\prime} i \not \partial \psi^{\prime}-g \int d x\left(\bar{\psi}^{\prime} \psi^{\prime}\right)^{2}+\text { const, }
$$

i.e. it reproduces $H^{I n_{0}}[z$ and $g$ are dimensionless and $\Gamma(x)=0$ at $x=0]$. For $k=0$, $\ell=2$, the graphs of Fig. 1 contribute, where the continuous lines indicate the flow of the flavor indices and the arrow the flow of charge: $\langle\zeta \widetilde{\zeta}\rangle \equiv \leftarrow$.

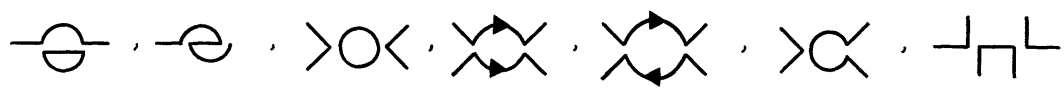

Fig. 1

For example, the two-point function graphs give

$$
\begin{aligned}
& -4 L^{3} g^{2} \int d x_{1} d x_{2}\left[N \bar{\psi}\left(x_{1}\right) \Gamma\left(L x_{1}-L x_{2}\right) \psi\left(x_{2}\right) \operatorname{tr} \Gamma\left(L x_{1}-L x_{2}\right)^{2}\right. \\
& \left.-\bar{\psi}\left(x_{1}\right) \Gamma\left(L x_{1}-L x_{2}\right)^{3} \psi\left(x_{2}\right)\right] .
\end{aligned}
$$

For later book-keeping purposes, it is convenient to normal order the terms of $H_{n_{0}-1}^{I n_{0}}$ with $m\left(=\sum m_{i}\right) \leqq 8$. For an arbitrary Hamiltonian $H(\tilde{\psi})$ and $G=\mathscr{G}_{1}$ of $(10)$,

$$
: H(\tilde{\psi}):=\exp \left[-\int d x_{1} d x_{2} \frac{\delta}{\delta \bar{\psi}\left(x_{1}\right)} G\left(x_{1}-x_{2}\right) \frac{\delta}{\delta \psi\left(x_{2}\right)}\right] H(\tilde{\psi}) \text {. }
$$

: $H$ : has the convenient property that $T$ acts on it as scaling in the linear order:

$$
\int d \mu_{\Gamma}(\widetilde{\zeta}): H\left(L^{-1 / 2} \tilde{\psi}\left(L^{-1} \cdot\right)+\widetilde{\zeta}\right):=: H\left(L^{-1 / 2} \tilde{\psi}\left(L^{-1} \cdot\right)\right): .
$$

Performing the normal ordering of the contributions of the graphs of Fig. 1 and using the identity $G(x)=L^{-1} G\left(L^{-1} x\right)+\Gamma(x)$, we obtain:

I. The quadratic term

$$
\begin{aligned}
& -4 g^{2} \int: N \bar{\psi}\left(x_{1}\right)\left[L G\left(L x_{1}-L x_{2}\right) \operatorname{tr} L^{2} G\left(L x_{1}-L x_{2}\right)^{2}-(L=1)\right] \psi\left(x_{2}\right) \\
& -\bar{\psi}\left(x_{1}\right)\left[L^{3} G\left(L x_{1}-L x_{2}\right)^{3}-(L=1)\right] \psi\left(x_{2}\right):,
\end{aligned}
$$

II. the quartic one

$$
\begin{aligned}
& -2 g^{2} \int: N \bar{\psi} \psi\left(x_{1}\right) \operatorname{tr}\left(L^{2} G\left(L x_{1}-L x_{2}\right)-(L=1)\right) \bar{\psi} \psi\left(x_{2}\right) \\
& \quad+\left[\left(\bar{\psi}\left(x_{1}\right) L G\left(L x_{1}-L x_{2}\right) \psi\left(x_{2}\right)\right)^{2}-(L=1)\right] \\
& \quad-\left[\left(\bar{\psi}\left(x_{1}\right) L G\left(L x_{1}-L x_{2}\right) \psi\left(x_{2}\right)\right)\left(\bar{\psi}\left(x_{2}\right) L G\left(L x_{1}-L x_{2}\right) \psi\left(x_{1}\right)\right)-(L=1)\right] \\
& \quad-2 \bar{\psi} \psi\left(x_{1}\right) \bar{\psi}\left(x_{2}\right)\left[L^{2} G\left(L x_{1}-L x_{2}\right)^{2}-(L=1)\right] \psi\left(x_{2}\right):,
\end{aligned}
$$

III. the sixth order one

$$
-4 g^{2} \int: \bar{\psi} \psi\left(x_{1}\right) \bar{\psi}\left(x_{1}\right)\left[L G\left(L x_{1}-L x_{2}\right)-(L=1)\right] \psi\left(x_{2}\right) \bar{\psi} \psi\left(x_{2}\right): .
$$


Next, we separate the dimensionless local parts of (41) and (42). We work directly in the position space without going to the momentum representation. This is somewhat unconventional but leads faster to the numerical results, exhibits better their universality and will leave us with convenient expressions for the remainders. First substitute into

$$
\int d x_{1} d x_{2}: \bar{\psi}\left(x_{1}\right) H^{2}\left(x_{1}-x_{2}\right) \psi\left(x_{2}\right):
$$

the decomposition

$$
\begin{aligned}
\psi\left(x_{2}\right)= & \psi\left(x_{1}\right)+\left(x_{2}-x_{1}\right) \partial \psi\left(x_{1}\right) \\
& +\int_{0}^{1} d t(1-t)\left(x_{2}-x_{1}\right)\left(x_{2}-x_{1}\right) \partial \partial \psi\left(x_{1}+t\left(x_{2}-x_{1}\right)\right)
\end{aligned}
$$

and notice that by the chiral symmetry the local $\bar{\psi} \psi$ term vanishes. Thus, after a simple change of variables $\left(s=t^{-1}\right),(44)$ becomes

$$
\begin{aligned}
& \int d x_{1}: \bar{\psi}\left(x_{1}\right)\left(\int d x_{2} H^{2}\left(x_{1}-x_{2}\right)\left(x_{2}-x_{1}\right)\right) \partial \psi\left(x_{1}\right): \\
& \quad-\int_{1}^{\infty} d s(s-1) \int d x_{1} d x_{2}: \partial \bar{\psi}\left(x_{1}\right)\left(x_{1}-x_{2}\right) H^{2}\left(s x_{1}-s x_{2}\right)\left(x_{1}-x_{2}\right) \partial \psi\left(x_{2}\right): .
\end{aligned}
$$

But for (44) representing (41),

$$
H^{2}(x)=L^{3} \tilde{H}^{2}(L x)-(L=1),
$$

so that

$$
\begin{aligned}
\int d x_{2} H^{2}\left(x_{1}-x_{2}\right)\left(x_{2}-x_{1}\right) & =-\int d x \int_{1}^{L} d \lambda \frac{d}{d \lambda} \lambda^{2} \tilde{H}^{2}(\lambda x) \lambda x \\
& =-\int_{1}^{L} \frac{d \lambda}{\lambda} \int d x\left[2 \tilde{H}^{2}(x) x+x \partial\left(\tilde{H}^{2}(x) x\right)\right] \\
& =-\log L \lim _{R \rightarrow \infty} \int_{|x|=R} d \sigma(x)|x| \tilde{H}^{2}(x) x,
\end{aligned}
$$

where we have used the Gauss theorem. Now, at long distances,

$$
G(x)=\frac{i x}{2 \pi x^{2}}+o\left(|x|^{-1}\right)
$$

(independently of the short distance regularization). Given (49), we easily compute from (41) the value of (48), and hence the local contribution to (41) given by the first term of (46) as

$$
\gamma_{2} g^{2} \log L \int d x: \bar{\psi} i \not \partial \psi:
$$

where

$$
\gamma_{2}=(2 N-1)\left(2 \pi^{2}\right)^{-1} \text {. }
$$

$\gamma_{2}$ is the first (universal) coefficient of the RG $\gamma$ function.

Similarly for the quartic term (42),

$$
\int d x_{1} d x_{2}: \bar{\psi} \psi\left(x_{1}\right) H^{4}\left(x_{1}-x_{2}\right) \bar{\psi} \psi\left(x_{2}\right):,
$$


by writing

$$
\bar{\psi} \psi\left(x_{2}\right)=\bar{\psi} \psi\left(x_{1}\right)+\int_{0}^{1} d t\left(x_{2}-x_{1}\right) \partial(\bar{\psi} \psi)\left(x_{1}+t\left(x_{2}-x_{1}\right)\right),
$$

we get the expression

$$
\begin{aligned}
& \int d x_{1}: \bar{\psi} \psi\left(x_{1}\right) \int d x H^{4}(x) \bar{\psi} \psi\left(x_{1}\right) \\
& \quad-\int_{1}^{\infty} d s \int d x_{1} d x_{2} \bar{\psi} \psi\left(x_{1}\right) H^{4}\left(x_{1}-x_{2}\right)\left(x_{2}-x_{1}\right) \partial(\bar{\psi} \psi)\left(x_{2}\right): .
\end{aligned}
$$

But

$$
H^{4}(x)=L^{2} \tilde{H}^{4}(L x)-(L=1)
$$

so that

$$
\int d x H^{4}(x)=\log L \lim _{R} \int_{|x|=R} d \sigma(x)|x| \tilde{H}^{4}(x),
$$

and (42) and (49) give immediately for the first, local term of (54)

$$
\beta_{2} g^{2} \log L \int d x:(\bar{\psi} \psi)^{2}:,
$$

with

$$
\beta_{2}=-\pi^{-1}(2 N-2),
$$

being the first (universal) coefficient of the $\mathrm{RG} \beta$ function. Note that for $N>1, \beta_{2}$ is negative, demonstrating the perturbative asymptotic freedom of the Gross-Neveu model.

B. Local Couplings: all Orders. Now simple generalizations of the procedures (46) and (54) of separation of the local contributions may be applied to any (normal ordered) $H^{\mathrm{mr}}$ term of (35) quadratic or quartic in the fields. For example for $m=4$, $m_{i}=1, r_{i}=0$ term, we use

$$
\prod_{i=1}^{4} \tilde{\psi}\left(x_{i}\right)=\prod_{i}\left(\tilde{\psi}\left(x_{1}\right)-\int_{0}^{1} d t\left(x_{i}-x_{1}\right) \partial \tilde{\psi}\left(x_{1}+t\left(x_{i}-x_{1}\right)\right)\right),
$$

which allows us to rewrite $\int d \mathbf{x} H^{\mathbf{m r}}(\mathbf{x}): \prod \tilde{\psi}\left(x_{i}\right)$ : as a local contribution

$$
\int d \mathbf{x} H^{\mathbf{m r}}(\mathbf{x}) \tilde{\psi}\left(x_{1}\right)^{4}
$$

and a combination of terms

$$
\int_{1}^{\infty} \prod_{n_{i}>0} d s_{i} s_{i} \int d \mathbf{x} H^{\mathrm{mr}}\left(x_{1}+s_{i}^{n_{i}}\left(x_{i}-x_{1}\right)\right): \prod_{1}^{4}\left(\left(x_{i}-x_{1}\right) \partial\right)^{n_{i}} \tilde{\psi}\left(x_{i}\right):,
$$

where $n_{1}=0, n_{2,3,4}=0,1$, and $\sum n_{i}>0$. It is crucial for renormalizability to know exactly the form of the local dimensionless terms. For the quadratic one, only $\bar{\psi} i \not \partial \psi$ is compatible with euclidean and chiral symmetries ( $T$ preserves these!). For quartic terms the situation is more subtle. Apart from $(\bar{\psi} \psi)^{2}$, also $\left(\psi \gamma^{\mu} \psi\right)^{2}$ and $\left(\bar{\psi} \gamma^{5} \psi\right)^{2}$ are allowed by the symmetries (these are all, see [3]).

We now show perturbatively that these terms are absent to all orders and for all $\tilde{\Lambda}$. Since in finite volume the kernels of $H_{\tilde{X}}^{\mathrm{EFF}}$ of $(30)$ are meromorphic in $z^{\Lambda}$ and 
$g^{\Lambda}$ (as follows from the bounds of the appendices), this settles the question once and for all: such terms will not emerge in the iteration. The absence of the unwanted quartic terms follows from an analogue of Furry's theorem. Consider graphs with four external lines. First we shall take graphs with $(\bar{\psi} \psi)^{2}$ vertices only. Following the flow of flavor and charge from the external lines these may be paired as in Fig. 2:
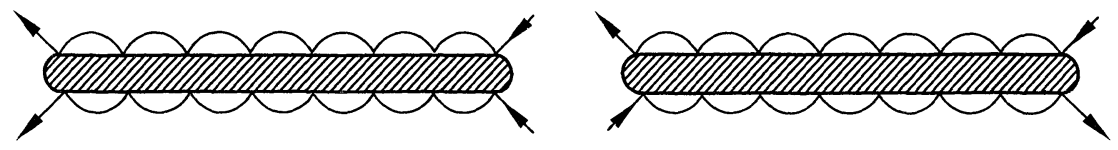

Fig. 2

giving

$$
\begin{aligned}
& \int d \mathbf{x} d \mathbf{y}\left[\bar{\psi}\left(x_{0}\right) \prod K_{i}\left(x_{i}-x_{i+1}\right) \psi\left(x_{p}\right)\right. \\
& \left.\quad+\bar{\psi}\left(x_{p}\right) \prod K_{p-i-1}\left(x_{p-i}-x_{p-i-1}\right) \psi\left(x_{0}\right)\right] \\
& \quad \cdot\left[\bar{\psi}\left(y_{0}\right) \prod L_{j}\left(y_{j}-y_{j+1}\right) \psi\left(y_{q}\right)\right. \\
& \left.\quad+\bar{\psi}\left(y_{q}\right) \prod L_{q-j-1}\left(y_{q-j}-y_{q-j-1}\right) \psi\left(y_{0}\right)\right] \tilde{H}(\mathbf{x}, \mathbf{y}),
\end{aligned}
$$

where $K_{i}(x-y)$ and $L_{j}(x-y)$ are either $\Gamma(L x-L y)$ or $G(x-y)$ (the latter due to the normal ordering). $\tilde{H}(\mathbf{x}, \mathbf{y})$ is a kernel. Note that if $C$ is a spinorial matrix such that

$$
\left(\gamma^{\mu}\right)^{T}=-C \gamma^{\mu} C^{-1}
$$

then, since $-K_{i}(x)=K_{i}(-x)$,

$$
\prod K_{p-i-1}\left(x_{p-i}-x_{p-i-1}\right)=\left[C \prod K_{i}\left(x_{i}-x_{i+1}\right) C^{-1}\right]^{T},
$$

and analogically for the $L$ 's.

But $\left[C \gamma^{5} C^{-1}\right]^{T}=-\gamma^{5}$ and $\left[C \gamma^{\mu} C^{-1}\right]^{T}=-\gamma^{\mu}$. Thus only the expressions $(\bar{\psi} \psi)^{2}$ will survive at zero momentum, the other contributions will cancel each other between the graphs of Fig. 2. The above argument can be easily extended to include the $\bar{\psi} i \not \partial \psi$ vertices. It also survives in the presence of a mass term in the original Hamiltonian which breaks the chiral invariance.

C. Operators of Higher Dimension. We have so far obtained, that after the normal ordering of terms with $m \leqq 8$ and extraction of the local contributions from the $m=2$ and $m=4$ ones, (35) can be written as (drop the superscript $n_{0}$ )

$$
\begin{aligned}
H_{n_{0}-1}^{I}(\tilde{\psi})= & \int\left(z_{n_{0}-1}: \bar{\psi} i \phi \psi:-g_{n_{0}-1}:(\bar{\psi} \psi)^{2}:\right) \\
& +\sum_{\mathbf{m}, \mathbf{r}, m \leqq 8} \int d \mathbf{x} H_{n_{0}-1}^{\mathbf{m r}}(\mathbf{x}): \prod_{1}^{q}\left(\partial \tilde{\psi}\left(x_{i}\right)\right)^{r_{i}}\left(\tilde{\psi}\left(x_{i}\right)\right)^{m_{i}-r_{i}} \\
& +\sum_{m>8} \int d \mathbf{x} H_{n_{0}-1}^{\mathbf{m r}}(\mathbf{x}) \prod(\partial \tilde{\psi})^{r_{i}} \tilde{\psi}^{m_{l}-r_{\imath}} .
\end{aligned}
$$

For $m=2, r_{i}=2$ and for $m=4, r_{i} \geqq 1$.

The wave function renormalization flows as

$$
\left|z_{n_{0}-1}-z-\gamma_{2} g^{2} \log L\right| \leqq \mathcal{O}\left(g^{3}\right),
$$

from (37) and (50). Due to the convergence of the expansion, (66) will become a rigorous bound. 
The flow of the coupling from (37) and (57) is

$$
\left|g_{n_{0}-1}-g+\beta_{2} g^{2} \log L\right| \leqq \mathcal{O}\left(g^{3}\right) \text {. }
$$

We need the $\mathcal{O}\left(g^{3}\right)$ terms exactly as well, to control the global flow. They come from $k=0, \ell=3$ terms and $k=1, \ell=2$ terms of (34). Denote them $b_{0} g^{3}$ and $c_{0} z g^{2}$ :

$$
\left|g_{n_{0}-1}-g+\beta_{2} g^{2} \log L-b_{0} g^{3}-c_{0} g^{2} z\right| \leqq \mathcal{O}\left(g^{4}\right) .
$$

For the terms of higher dimension in (65) we also separate the leading perturbative parts explicitly:

$H^{m r}$ with $m=(2,2), r=(0,1)$ (and permutations thereof). We write

$$
H_{n_{0}-1}^{\mathrm{mr}}\left(x_{1}, x_{2}\right)=g_{n_{0}-1}^{2} L_{1}\left(x_{1}, x_{2}\right)+\tilde{H}_{n_{0}-1}^{\mathrm{mr}}\left(x_{1}, x_{2}\right),
$$

where the $L_{1}$ term, modulo the change $g^{2} \rightarrow g_{n_{0}-1}^{2}$ introducing an $\mathcal{O}\left(g^{3}\right)$ error absorbed into $\tilde{H}$, arises from the second order perturbative contribution (42) as a kernel of the non-local term in (54).

$H^{\mathbf{m r}}$ with $\mathbf{m}=(1,3), \mathbf{r}=(1,0)$. We take out the contribution of the $k=l=1$ term of (34) given by the graph $\longrightarrow\left\ulcorner\right.$, modulo the change $z g \mapsto z_{n_{0}-1} g_{n_{0}-1}$ :

$$
H_{n_{0}-1}^{\mathrm{mr}}\left(x_{1}, x_{2}\right)=z_{n_{0}-1} g_{n_{0}-1} K_{1}\left(x_{1}, x_{2}\right)+\tilde{H}_{n_{0}-1}^{\mathbf{m r}}\left(x_{1}, x_{2}\right) \text {. }
$$

$H^{m r}$ with $m=(3,3), r=(0,0)$.

$$
H_{n_{0}-1}^{\mathrm{mr}}\left(x_{1}, x_{2}\right)=g_{n_{0}-1}^{2} M_{1}\left(x_{1}, x_{2}\right)+\tilde{H}_{n_{0}-1}^{\mathrm{mr}}\left(x_{1}, x_{2}\right),
$$

where the $M_{1}$ term comes from (43).

All our kernels are given in terms of connected graphs with lines $\Gamma$ decaying exponentially. This and the convergence of this graphical expansion will allow us to prove in the next section bounds which we shall state now. We denote $[$ see $(25)]$

$$
\left\|H_{n_{0}-1}^{m}\right\|=\sum_{\mathbf{m}, \mathbf{r}}\left\|H_{n_{0}-1}^{\mathbf{m r}}\right\|
$$

and for $m=4$ or 6 ,

$$
\left\|\tilde{H}_{n_{0}-1}^{m}\right\|=\sum\left\|\tilde{H}_{n_{0}-1}^{\mathrm{mr}}\right\|,
$$

where $\tilde{H}_{n_{0}-1}^{\mathrm{mr}}=H_{n_{0}-1}^{\mathrm{mr}}$ except the cases listed above. In this notation,

$$
\begin{gathered}
\left\|H_{n_{0}-1}^{2}\right\| \leqq A^{2} g_{n_{0}-1}^{2}, \\
\left\|\tilde{H}_{n_{0}-1}^{m}\right\| \leqq A^{m} g_{n_{0}-1}^{3} \quad \text { for } \quad m=4,6, \\
\left\|H_{n_{0}-1}^{m}\right\| \leqq A^{m} g_{n_{0}-1}^{3+\varepsilon(m-8)}, \quad m \geqq 8 .
\end{gathered}
$$

Perturbatively, the $g$-dependence in (74)-(76) is obvious. In fact, one has (76) with $\varepsilon=\frac{1}{2}$, the worst contributions coming from the graphs of Fig. 3. It is enough, and convenient, to take $\varepsilon$ smaller, say $\frac{1}{10}$. $A$ is a suitably chosen constant, as is $C_{1}$ in (72) and $C_{i}$ below.

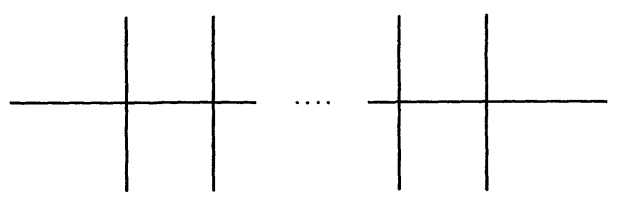


D. Iterating the $R G$. Now consider iterating the above, i.e. applying $T$ to $H_{n_{0}-1}^{I}$ as given by (65). The crucial point is that again we may proceed perturbatively. Indeed, generally

$$
H_{n-1}^{I}\left(\tilde{\psi}^{\prime}\right)=-\sum_{k=1}^{\infty} \frac{(-)^{k}}{k !}\left\langle H_{n}^{I}\left(L^{-1 / 2} \tilde{\psi}\left(L^{-1} \cdot\right)+\tilde{\zeta}^{k}\right\rangle_{\Gamma}^{T},\right.
$$

from which a representation like (65) with $n_{0} \rightarrow n$ emerges if $H_{n}^{I}$ has one. Here we shall only state the estimates and see the general structure of the terms which arises in the iteration. The proof establishing the convergence of expansions (77) will be discussed in the next section.

The Wave Function Renormalization. Picking the lowest perturbative contributions as in (66) and (67), we get for $z_{n}$ and $g_{n}$

$$
\begin{aligned}
& \left|z_{n-1}-z_{n}-\gamma_{2} g_{n}^{2} \log L\right| \leqq C_{2} g_{n}^{3}, \\
& \left|g_{n-1}-g_{n}+\beta_{2} g_{n}^{2} \log L\right| \leqq C_{3} g_{n}^{3} .
\end{aligned}
$$

Expressions (78) and (79) allow us to trace (iteratively) the behavior of the wave function renormalization. Namely, we get

$$
\left|z_{n}+\frac{\gamma_{2}}{\beta_{2}} g_{n}\right| \leqq C_{4} g_{n}^{2}
$$

Indeed,

$$
\begin{aligned}
\left|z_{n-1}+\frac{\gamma_{2}}{\beta_{2}} g_{n-1}\right| & \leqq\left|z_{n}+\frac{\gamma_{2}}{\beta_{2}} g_{n}+\gamma_{2} g_{n}^{2} \log L-\frac{\gamma_{2}}{\beta_{2}} \beta_{2} g_{n}^{2} \log L\right|+\left(C_{2}+C_{3}\right) g_{n}^{3} \\
& \leqq C_{4} g_{n}^{2}+\left(C_{2}+C_{3}\right) g_{n}^{3} .
\end{aligned}
$$

But $g_{n}$ increases: $g_{n}^{2}=g_{n-1}^{2}-2\left|\beta_{2}\right| g_{n}^{3} \log L+\mathcal{O}\left(g_{n}^{4}\right)$, thus (81) yields (80) with $n-1$, if $C_{4}$ is large enough $\left(C_{2}\right.$ and $C_{3}$ don't depend on $\left.C_{4}\right)$.

$H_{n}^{\mathrm{mr}}$ with $m=2$. Since it enters into an irrelevant term, the bound (74) for it easily iterates

$$
\left\|H_{n}^{2}\right\| \leqq A^{2} g_{n}^{2} .
$$

$H_{n}^{\mathbf{m r}}$ with $\mathbf{m}=(2,3), \mathbf{r}=(0,1)$. We have

$$
H_{n}^{\mathrm{mr}}\left(x_{1}, x_{2}\right)=g_{n}^{2} L_{n_{0}-n}\left(x_{1}, x_{2}\right)+\tilde{H}_{n}^{\mathrm{mr}}\left(x_{1}, x_{2}\right),
$$

where

$$
L_{n_{0}-n}\left(x_{1}, x_{2}\right)=\sum_{\ell=0}^{n_{0}-n-1} L^{\ell} L_{1}\left(L^{\ell} x_{1}, L^{\ell} x_{2}\right) .
$$

The $L_{n_{0}-n}$ term collects the perturbative $\mathcal{O}\left(g_{n}^{2}\right)$ contributions (at each step the old term picks powers of $L$, new $L_{1}$ term is created and the coupling constant is updated by delegating the error to $\widetilde{H}$ ).

Similarly, for $\mathbf{m}=(1,3), \mathbf{r}=(1,0)$,

$$
H_{n}^{\mathrm{mr}}(\mathbf{x})=z_{n} g_{n} K_{n_{0}-n}(\mathbf{x})+\tilde{H}_{n}^{\mathrm{mr}}(\mathbf{x}),
$$


with

$$
K_{n_{0}-n}(\mathbf{x})=\sum_{\ell=0}^{n_{0}-n-1} L^{\ell} K_{1}\left(L^{\ell} \mathbf{x}\right)
$$

and for $\mathbf{m}=(3,3), \mathbf{r}=(0,0)$

$$
H_{n}^{\mathrm{mr}}(\mathbf{x})=g_{n}^{2} M_{n_{0}-n}(\mathbf{x})+\tilde{H}_{n}^{\mathrm{mr}}(\mathbf{x}),
$$

with

$$
M_{n_{0}-n}=\sum_{0}^{n_{0}-n-1} L^{\ell} M_{1}\left(L^{\ell} \mathbf{x}\right)
$$

Since $\left\|L_{1}\right\|,\left\|K_{1}\right\|,\left\|M_{1}\right\|$ are finite and

$$
\left\|L_{n_{0}-n+1}-L_{n_{0}-n}\right\| \leqq L^{n_{0}-n}\left\|L_{1}\right\|,
$$

it follows that

$$
\begin{gathered}
L_{n_{0}-n} \rightarrow L_{*}, \\
\left\|L_{*}-L_{n_{0}-n}\right\| \leqq\left(1-L^{-1}\right)^{-1} L^{n_{0}-n}\left\|L_{1}\right\|,
\end{gathered}
$$

and the same for $K$ and $M$. For the remainders, (75) iterates:

$$
\left\|\tilde{H}_{n}^{m}\right\| \leqq A^{m} g_{n}^{3}, \quad m=4,6 .
$$

Finally, as in (76)

$$
\left\|H_{n}^{m}\right\| \leqq A^{m} g_{n}^{3+\varepsilon(m-8)}, \quad m \geqq 8 .
$$

We end this section by showing how the $\beta$-function (flow of $g_{n}$ ) stabilizes to third order and why this suffices for the global control of the flow. The $\mathcal{O}\left(g_{n}^{3}\right)$ terms of the recursion of $g_{n}$ are besides the contributions $-b_{0} g_{n}^{3}$ and $-c_{0} z_{n} g_{n}^{2}$ from the $z_{n}$ and $g_{n}$ vertices [compare (68)], also the term $\tilde{b}_{n_{0}-n} g_{n}^{3}$ linear in $L_{n_{0}-n}$ and $M_{n_{0}-n}$ and the term $\widetilde{C}_{n_{0}-n} z_{n} g_{n}^{2}$ linear in $K_{n_{0}-n}$ and $M_{n_{0}-n}$ [the fact that the $K, L, M \mathscr{O}\left(g_{n}^{2}\right)$ nonlocal terms of $H_{n}^{I}$ contribute at the third order to $g_{n-1}$ was the reason for the special treatment they obtained; notice that the non-local quadratic term, also $\mathcal{O}\left(g_{n}^{2}\right)$, does not!]. We shall get

$$
\begin{aligned}
& \left|g_{n-1}-g_{n}+\beta_{2} g_{n}^{2} \log L-\left(b_{0}+\tilde{b}_{n_{0}-n}\right) g_{n}^{3}-\left(c_{0}+\tilde{c}_{n_{0}-n}\right) g_{n}^{2} z_{n}\right| \\
& \quad \leqq C_{5} g_{n}^{3+2 \varepsilon} .
\end{aligned}
$$

Note that due to (89) and its analogues for $K$ and $M$,

$$
\left|\tilde{b}_{n_{0}-n+1}-\tilde{b}_{n_{0}-n}\right|,\left|\tilde{c}_{n_{0}-n+1}-\tilde{c}_{n_{0}-n}\right| \leqq C L^{n-n_{0}} .
$$

Expressions (80) and (95) imply that the coefficient at $g_{n}^{3}$ in the $g_{n}$ recursion

$$
B_{n_{0}-n} \equiv-b_{0}-\tilde{b}_{n_{0}-n}+\frac{\gamma_{2}}{\beta_{2}}\left(c_{0}+\tilde{c}_{n_{0}-n}\right)
$$

stabilizes exponentially fast to a value $B_{*}$. $B_{*}$ is related to the $\beta_{3}$ coefficient of the $\mathrm{RG} \beta$-function appearing in (21) through

$$
\beta_{3}=\left(B_{*}+\beta_{2}^{2}(\log L)^{2}\right) / \log L
$$


(the right-hand side is $L$-independent as $\beta_{3}$ is universal, but we shall not need it).

Let us show iteratively that (94) is solved by

$$
\left|g_{n}-g^{n}\right| \leqq C\left(g^{n}\right)^{2},
$$

where $g^{n}$ is given by (21) (with $\Lambda=L^{n}$ ). Indeed, we show equivalently that

$$
\left|g_{n}^{-1}-\left(g^{n}\right)^{-1}\right| \leqq C^{\prime}
$$

Namely, from (21), (94), and (96),

$$
\begin{aligned}
\left|g_{n-1}^{-1}-\left(g^{n-1}\right)^{-1}\right| \leqq & C^{\prime}+\left|g_{n-1}^{-1}-g_{n}^{-1}-\left(g^{n-1}\right)^{-1}+\left(g^{n}\right)^{-1}\right| \\
\leqq & C^{\prime}+\mid \beta_{2} \log L+\left(\beta_{2} \log L\right)^{2} g_{n}+B_{n_{0}-n} g_{n}+\mathcal{O}\left(g_{n}^{2}\right) \\
& -\beta_{2} \log L-\beta_{3} g_{n} \log L+\mathcal{O}\left(\left(g^{n}\right)^{2}\left|\log g^{n}\right|\right) \mid \\
\leqq & C^{\prime}+\mathcal{O}\left(L^{n-n_{0}} \frac{1}{n}\right)+\mathcal{O}\left(\frac{\log n}{n^{2}}\right) .
\end{aligned}
$$

The right-hand side is summable in $n$, hence (99) is true.

Now we turn to the derivation of the bounds formulated above.

\section{Convergence of the Iterative Perturbation Expansion}

We have to iterate the data for $H_{n}$, the effective Hamiltonian on scale $L^{n}$. Let us start by rewriting the representation (65) (with $n_{0}-1$ replaced by $n$ ) in a more compact notation. We shall include $\tilde{\psi}(x)$ and $\partial \tilde{\psi}(x)$ into the same multiplet denoted by $\left(\Psi_{A}(x)\right)$. It has $12 N$ components indicated by subscript $A$. Equation (65) becomes

$$
\begin{aligned}
H_{n}^{I}(\Psi)= & \sum_{m \leqq 8} \sum_{\mathbf{A}} \int d \mathbf{x} H_{n}^{m}(\mathbf{x}, \mathbf{A}): \Psi(\mathbf{x}, \mathbf{A}): \\
& +\sum_{m>8} \sum_{\mathbf{A}} \int d \mathbf{x} H_{n}^{m}(\mathbf{x}, \mathbf{A}) \Psi(\mathbf{x}, \mathbf{A}),
\end{aligned}
$$

with $H_{n}^{m}(\mathbf{x}, \mathbf{A})$ totally anti-symmetric under $\left(x_{k}, A_{k}\right) \leftrightarrow\left(x_{j}, A_{j}\right)$ and including $\delta$-functions to produce the powers of fields in the following way:

$$
H_{n}^{m}(\mathbf{x}, \mathbf{A})=\mathscr{A}\left(\sum_{\mathbf{m}, \mathbf{r}} H_{n}^{\mathbf{m r}}\left(x_{m_{1}^{\prime}} \ldots x_{m_{q^{\prime}}}, \mathbf{A}\right) \prod_{i=1}^{q} \prod_{j=m_{1-1}^{\prime}+1}^{m_{i}-1} \delta\left(x_{j}-x_{m_{i}^{\prime}}\right)\right) .
$$

In (102), $H_{n}^{\mathrm{mr}}$ are local and non-local terms of (65) with $m_{i}^{\prime}=m_{1}+\ldots+m_{i}, H_{n}^{\mathrm{mr}}(\mathbf{x}, \mathbf{A})$ is nonzero only if $\mathbf{A} \equiv\left(A_{1}, \ldots, A_{m}\right)$ contains the derivative indices in appropriate places specified by $\mathbf{r} . \mathscr{A}$ is the antisymmetrizer in $\left\{\left(x_{i}, A_{i}\right)\right\}$ :

$$
\mathscr{A}(F(\mathbf{x}, \mathbf{A}))=(m !)^{-1} \sum_{\pi \in S_{m}}(-)^{\pi} F\left(x_{\pi(1)}, A_{\pi(1)}, \ldots, x_{\pi(m)}, A_{\pi(m)}\right) .
$$

$H_{n}^{m}$ 's are no more functions but measures. The norm

$$
\left\|H_{n}^{m}\right\|=\sum_{\mathbf{A}} \int d x_{2} \ldots d x_{m}\left|H_{n}^{m}(\mathbf{x}, \mathbf{A})\right| e^{\mathscr{L}(\mathbf{x})},
$$

still makes sense and the patient reader will check that with our index conventions,

$$
\left\|H_{n}^{m}\right\|=\sum_{\mathbf{m}, \mathbf{r}}\left\|H_{n}^{\mathbf{m r}}\right\|
$$

[cf. (25)], so that (104) is consistent with (72). 
Let us derive the recursion between $H_{n}$ and $H_{n-1}$. We compute perturbatively

$$
\begin{aligned}
H_{n-1}^{m}(\mathbf{x}, \mathbf{A}) & =-(m !)^{-1} \frac{\delta}{\delta \Psi_{A_{m}}\left(x_{m}\right)} \cdots \frac{\delta}{\delta \Psi_{A_{1}}\left(x_{1}\right)} \uparrow_{\Psi=0} \log \int e^{-H_{n}^{I}\left(\Psi_{L}+Z\right)} d \mu_{\Gamma}(\widetilde{\zeta}) \\
& =\frac{1}{m !} \sum_{\left\{I_{\alpha}\right\}} \sum_{\ell=0}^{\infty} \frac{(-)^{\#}}{\ell !}\left\langle\prod_{\alpha=1}^{k} \frac{\delta^{m_{\alpha}} H_{n}^{I}}{\delta \Psi\left(\mathbf{x}_{\alpha}, \mathbf{A}_{\alpha}\right)} \prod_{\alpha=k+1}^{k+\ell} H_{n}^{I}\right\rangle_{\Gamma}^{T} \Gamma_{\Psi=0}
\end{aligned}
$$

where $\left\{I_{\alpha}\right\}_{1}^{k}$ is a partition of $\{1, \ldots, m\}, m_{\alpha}=\left|I_{\alpha}\right|,(-1)^{\#}$ is a sign which we do not have to keep track of, $\frac{\delta^{m_{\alpha}}}{\delta \Psi\left(\mathbf{x}_{\alpha}, \mathbf{A}_{\alpha}\right)}=\prod_{i \in I_{\alpha}} \frac{\delta}{\delta \Psi_{A_{i}}\left(x_{i}\right)}$,

and

$$
\Psi_{L}(x)=\left(L^{-1 / 2} \tilde{\psi}(x / L), L^{-3 / 2}(\partial \tilde{\psi})(x / L)\right), \quad Z=(\tilde{\zeta}, \partial \tilde{\zeta}) .
$$

Let us, for the time being undo the Wick ordering of $m \leqq 8$ terms. Since the covariance $G$ is bounded, we still have (using the same symbols and for $A$ big enough)

$$
\left\|H_{n}^{2}\right\| \leqq A^{2} g_{n}, \quad\left\|H_{n}^{4}\right\| \leqq A^{4} g_{n}, \quad\left\|H_{n}^{6}\right\| \leqq A^{6} g_{n}^{2},
$$

if the bounds described in the previous section hold for $H_{n}$. The kernels $H_{n}^{m}, m \geqq 8$, do not change and are bounded by (93). Now

$$
\begin{aligned}
\frac{\delta^{m_{\alpha}} H_{n}^{I}}{\delta \Psi\left(\mathbf{x}_{\alpha}, \mathbf{A}_{\alpha}\right)} \uparrow \Psi=0 & L^{\frac{3}{2} m_{\alpha}-r\left(\mathbf{A}_{\alpha}\right)} \sum_{s_{\alpha} \geqq m_{\alpha}}(-)^{\#} \frac{s_{a} !}{\left(s_{\alpha}-m_{\alpha}\right) !} \\
& \cdot \sum_{\mathbf{B}_{\alpha}} \int d \mathbf{y}_{\alpha} H_{n}^{s_{\alpha}}\left(L \mathbf{x}_{\alpha}, \mathbf{y}_{\alpha}, \mathbf{A}_{\alpha}, \mathbf{B}_{\alpha}\right) Z\left(\mathbf{y}_{\alpha}, \mathbf{B}_{\alpha}\right),
\end{aligned}
$$

where $r\left(\mathbf{A}_{\alpha}\right)$ is the number of indices in $\mathbf{A}_{\alpha}$, referring to $\partial \tilde{\psi}$ components of $\Psi$. Thus

$$
\begin{aligned}
H_{n-1}^{m}(\mathbf{x}, \mathbf{A})= & \frac{1}{m !} L^{-\frac{m}{2}-\mathbf{r}(\mathbf{A})} \sum_{I_{\alpha}, \ell, s_{\alpha}} L^{2 s} \frac{(-)^{\#}}{\ell !} \prod_{\alpha=1}^{k+\ell} \frac{s_{\alpha} !}{\left(s_{\alpha}-m_{\alpha}\right) !} \\
& \cdot \sum_{\mathbf{B}} \int d \mathbf{y} \prod_{\alpha} H_{n}^{s_{\alpha}}\left(L \mathbf{x}_{\alpha}, L \mathbf{y}_{\alpha}, \mathbf{A}_{\alpha}, \mathbf{B}_{\alpha}\right)\left\langle\prod_{\alpha} Z\left(L \mathbf{y}_{\alpha}, \mathbf{B}_{\alpha}\right)\right\rangle_{\Gamma}^{T},
\end{aligned}
$$

where $s=\sum s_{\alpha}, m_{\alpha}=0$ for $k<\alpha \leqq k+l, \mathbf{B}=\left(\mathbf{B}_{\alpha}\right)_{1}^{k+l}$, and $\mathbf{y}=\left(\mathbf{y}_{\alpha}\right)_{1}^{k+l}$.

To bound (109), let us separate first the leading $k=1, l=0, s=m$ term:

$$
H_{n-1}^{m}(\mathbf{x}, \mathbf{A})=L^{\frac{3}{2} m-r(\mathbf{A})} H_{n}^{m}(L \mathbf{x}, \mathbf{A})+H_{n-1}^{\prime m}(\mathbf{x}, \mathbf{A}) .
$$

Obviously [see (25)]

$$
L^{\frac{3}{2} m-r(\mathbf{A})} \int_{x_{1}=0} d \mathbf{x}\left|H_{n}^{m}(L \mathbf{x}, \mathbf{A})\right| e^{\mathscr{L}(\mathbf{x})} \leqq L^{2-\frac{m}{2}-r(\mathbf{A})}\left\|H_{n}^{m}\right\|,
$$

i.e. for $m>4$ or $m=4, r(\mathbf{A}) \geqq 1$ or $m=2, r(\mathbf{A})=2$, the leading term contracts. This contraction of the irrelevant terms, together with the convergence of (109) is, besides the behavior of the running coupling constant and the running wave function renormalization analysed in Sect. 5, the main mechanism behind the iteration of our bounds.

For the convergence of (109), the crucial role is played by the following estimate for the fluctuation Green function:

$$
\left|\left\langle\prod_{\alpha} Z\left(L \mathbf{y}_{\alpha}, \mathbf{B}_{\alpha}\right)\right\rangle_{\Gamma}^{T}\right| \leqq \prod_{j} N_{j} ! \exp \left[-L \mathscr{L}\left(\mathbf{y}_{1} ; \ldots ; \mathbf{y}_{k+\ell}\right)\right] \prod_{\alpha} C^{s_{\alpha}-m_{\alpha}} e^{\frac{L}{2} \mathscr{L}\left(\mathbf{y}_{\alpha}\right)} .
$$


The $N_{j}$ are as follows. Cover $\mathbb{R}^{2}$ by unit cubes. Let $X_{\alpha}$ be the union of cubes containing the points $L \mathbf{y}_{\alpha}$. Then $N_{j}$ are the multiplicities of occurrence of different sets in the collection. Roughly, $N_{j}$ measure number of similar $Z\left(L \mathbf{y}_{\alpha}\right)$ as far as their spatial location is concerned. $L\left(\mathbf{y}_{1} ; \ldots ; \mathbf{y}_{k+l}\right)$ is the length of the shortest graph on points of $\mathbf{y}_{\alpha}$ and possibly other points, connected once $\mathbf{y}_{\alpha}$ are shrunk to points. Inequality (112) is the typical behavior for the bounded spin systems in high temperature confirming boundedness of fermions [12]. Since its proof is slightly tedious, we defer it to appendices.

Given (112), it is an easy task to bound the expansion (109). Insertion of (112) to (109) leads to

$$
\begin{aligned}
\left\|H_{n-1}^{\prime m}\right\| \leqq & \frac{1}{m !} L^{-\frac{m}{2}} \sum_{\left\{I_{\alpha}\right\}, \ell,\left(s_{\alpha}\right), \mathbf{A}, \mathbf{B}}(\ell !)^{-1} L^{2 s} C^{s-m} \int_{x_{1}=0} d \mathbf{x} d \mathbf{y} \\
& \cdot \prod_{\alpha}\left(\frac{s_{\alpha} !}{\left(s_{\alpha}-m_{\alpha}\right) !}\left|H_{n}^{s_{\alpha}}\left(L \mathbf{x}_{\alpha}, L \mathbf{y}_{\alpha}, \mathbf{A}_{\alpha}, \mathbf{B}_{\alpha}\right)\right| e^{L \mathscr{L}\left(\mathbf{x}_{\alpha}, \mathbf{y}_{\alpha}\right)}\right) K,
\end{aligned}
$$

where now $s_{\alpha}>m_{\alpha}$ for each $\alpha$ and

$$
K=\prod_{j} N_{j} ! \exp \left[\mathscr{L}(\mathbf{x})+\frac{L}{2} \sum_{\alpha} \mathscr{L}\left(\mathbf{y}_{\alpha}\right)-L \mathscr{L}\left(\mathbf{y}_{1} ; \ldots ; \mathbf{y}_{k+\ell}\right)-L \sum_{\alpha} \mathscr{L}\left(\mathbf{x}_{\alpha}, \mathbf{y}_{\alpha}\right)\right] .
$$

Note the connected structure of (114). Due to the extra $L$ (resulting from scaling) in the negative terms, we have

$$
K \leqq \prod N_{j} ! e^{-\mathscr{L}(\mathbf{y})} .
$$

$\Pi N_{j}$ ! is eliminated as follows. Let $S=\left(y_{1}, \ldots, y_{k+\ell}\right)$ with $y_{\alpha}$ in $\mathbf{y}_{\alpha}$. Clearly

$$
\prod_{j} N_{j} ! \leqq \sum_{S} \prod_{i} M_{i} !
$$

where $M_{i}$ are the multiplicities of different unit cubes where $y_{i}$ lie. Also

$$
\mathscr{L}(\mathbf{y}) \geqq \mathscr{L}(S) \geqq \varepsilon \tilde{\mathscr{L}}(S),
$$

where $\tilde{\mathscr{L}}(S)$ is the length of the shortest tree on $S$ and no other points (in fact one can take $\varepsilon=1 / 2$ ). But now

$$
e^{-\varepsilon \tilde{\mathscr{L}}(\mathrm{S})} \leqq C^{k+\ell} \prod_{i} M_{i}^{2-M_{i}} \sum_{\tau} e^{-\varepsilon \mathscr{L}_{\tau}(S)},
$$

with $\tau$ running through all trees on $S$ and $\mathscr{L}_{\tau}(S)$ being the length of $\tau$. Inequality (117) with $C=1$ would follow if $M_{i}$ 's were multiplicities of $y_{\alpha}^{\text {'s }}$ as $M_{i}^{M_{2}-2}$ is the number of trees on $M_{i}$ vertices. $C^{k+\ell}$ is the fine for making groups of $M_{i} y_{\alpha}$ 's coincide. Equations (115) to (117) give

$$
K \leqq C^{k+\ell} \sum_{\tau} e^{-\varepsilon \mathscr{L}_{\tau}(\boldsymbol{S})} .
$$

Upon insertion of (118) into (113), we may perform the integrals in the order indicated by $\tau$, successively trimming its outmost branches:

$$
\begin{aligned}
& \sum_{\mathbf{A}, \mathbf{B}} \int d \mathbf{x} d \mathbf{y} \prod_{\alpha}\left|H_{n}^{s_{\alpha}}\left(L \mathbf{x}_{\alpha} \ldots \mathbf{B}_{\alpha}\right)\right| e^{L \mathscr{L}\left(\mathbf{x}_{\alpha}, \mathbf{y}_{\alpha}\right)} e^{-\varepsilon_{\mathscr{L}_{\tau}}(S)} \\
& \quad \leqq L^{-2 s} C^{k+\ell} \prod_{\alpha}\left\|H_{n}^{s_{\alpha}}\right\| .
\end{aligned}
$$


This gives

$$
\left\|H_{n-1}^{\prime m}\right\| \leqq \frac{1}{m !} L^{-\frac{m}{2}} \sum_{\left\{I_{\alpha}\right\} \ldots \tau}(\ell !)^{-1} C^{s-m} \prod_{\alpha} \frac{s_{\alpha} !}{\left(s_{\alpha}-m_{\alpha}\right) !}\left\|H_{n}^{s_{\alpha}}\right\|,
$$

where we also used the fact that $s-m \geqq k+\ell$. As

$$
\sum_{s, \tau} 1 \leqq 2^{s-m}(k+\ell)^{k+\ell-2} \leqq C^{s-m} k ! \ell !,
$$

and

$$
\begin{gathered}
\sum_{\left\{I_{\alpha}\right\}}=\sum_{\left(m_{\alpha}\right)} \frac{1}{k !} \frac{m !}{\prod m_{\alpha} !}, \\
\left\|H_{n-1}^{\prime m}\right\| \leqq L^{-\frac{m}{2}} \sum_{\left(m_{\alpha}\right), \ell,\left(s_{\alpha}\right)} 2^{s} C^{s-m} \prod_{\alpha}\left\|H_{n}^{s_{\alpha}}\right\| .
\end{gathered}
$$

Recall that $\left\|H_{n}^{s_{\alpha}}\right\|$ satisfy (107) or (93). Let us first consider the case $m>8$, where no low order analysis is needed. For $\varepsilon$ small, it is easy to check that if $m>8$, then

$$
\prod_{\alpha}\left\|H_{n}^{s_{\alpha}}\right\| \leqq A^{s} g_{n}^{3+\varepsilon(m-8)} g_{n}^{\varepsilon \Sigma\left(s_{\alpha}-m_{\alpha}\right)}
$$

Thus

$$
\begin{aligned}
\left\|H_{n-1}^{m}\right\| & \leqq 2^{m} L^{-\frac{m}{2}} A^{m} g_{n}^{3+\varepsilon(m-8)} \sum_{\left(m_{\alpha}\right), \ell} \prod_{\alpha}\left(\sum_{s_{\alpha}>m_{\alpha}}\left(2 A C g^{\varepsilon}\right)^{s_{\alpha}-m_{\alpha}}\right) \\
& \leqq A^{m} g_{n}^{3+\varepsilon(m-8)+\varepsilon} .
\end{aligned}
$$

Equations (110), (111), and (125) yield (93) for $m>8$.

For $m \leqq 8$, we need to extract some low order perturbative terms. Returning to the normal ordered expressions, we see that $k=1, \ell=0, m$ whatever terms of (109) give for $m<8$

$$
\left[H_{n-1}^{m}\right]_{\substack{k=1 \\ \ell=0}}=L^{\frac{3}{2} m-r(\mathbf{A})} H_{n}^{m}(L \mathbf{x}, \mathbf{A})+\mathcal{O}\left(g^{3+2 \varepsilon}\right),
$$

the last contribution coming from $s>8$ terms. For the rest, separate the expressions discussed in Sect. 5. For $m=8$,

$$
\left\|H_{n-1}^{8}\right\| \leqq L^{-2} A^{8} g_{n}^{3}+D g_{n}^{3}+\mathcal{O}\left(g_{n}^{3+2 \varepsilon}\right),
$$

where $D g_{n}^{3}$ estimates the contributions of the graphs involving three local fourpoint vertices or one four-point and one six-point expression, the latter proportional to $M_{n}$. For $A$ large, (93) iterates.

$H_{n-1}^{6}$ is similar. Separate the second order contribution $g_{n}^{2} M_{1}$ to the $M$ part of it, write

$$
M_{n_{0}-n+1}\left(x_{1}, x_{2}\right)=M_{1}\left(x_{1}, x_{2}\right)+L M_{n_{0}-n}\left(L x_{1}, L x_{2}\right),
$$

change $g_{n}^{2}$ in front of it to $g_{n-1}^{2}$ [anticipating that $g_{n-1}^{2}=g_{n}^{2}-2 \beta_{2} g_{n}^{3} \log L$ $\left.+\mathcal{O}\left(g_{n}^{4}\right)\right]$, and estimate

$$
\left\|\tilde{H}_{n-1}^{6}\right\| \leqq L^{-1}\left\|\tilde{H}_{n}^{6}\right\|+E g_{n}^{3}+F z_{n} g_{n}+\mathcal{O}\left(g_{n}^{3+2 \varepsilon}\right),
$$

which implies that (92) for $m=6$ iterates. 
Finally, to get $\tilde{H}_{n-1}^{4}$ and $H_{n-1}^{2}$, we have to separate the local contributions. We have (in a sloppy notation omitting $\delta$-functions, indices, and anti-symmetrizers)

$$
\begin{aligned}
H_{n-1}^{4}(\mathbf{x})= & g_{n}+g_{n}^{2} I_{1}\left(x_{2}, x_{4}\right)+g_{n}^{2} L L_{n_{0}-n}\left(L x_{2}, L x_{4}\right) \\
& +z_{n} g_{n} K_{1}\left(x_{1}, x_{4}\right)+z_{n} g_{n} L K_{n_{0}-n}\left(L x_{1}, L x_{4}\right)+L^{5} \tilde{H}_{n}^{4}(L \mathbf{x}) \\
& +g_{n}^{3} P_{n}(\mathbf{x})+z_{n} g_{n}^{2} Q_{n}(\mathbf{x})+z_{n}^{2} g_{n} R_{n}(\mathbf{x})+\mathcal{O}\left(g_{n}^{3+2 \varepsilon}\right),
\end{aligned}
$$

where $I_{1}$ term is (42) and $P_{n}, Q_{n}, R_{n}$ stabilize exponentially fast with $n_{0}-n$. Note how (126) picked $L^{5}$ instead of $L^{6}$ because $r(\mathbf{A}) \geqq 1$ in (126). Extract the local part as described in Sect. 5. This gives (94). The remainders can be collected into $g_{n-1}^{2} L_{n_{0}-n+1}, g_{n-1}^{2} K_{n_{0}-n-1}$, and $\tilde{H}_{n-1}^{4}$. Notice that the extraction of local terms does not change the $\|\cdot\|$ norm considerably. For example for the term of $(61)$, we get

$$
\int_{x_{1}=0} d \mathbf{x} \prod_{n_{i}>0}\left(\int_{1}^{\infty} d s_{i} s_{i}\left|x_{i}\right|\right)\left|H^{\mathbf{m r}}\left(s^{n_{j}} x_{j}\right)\right| e^{\mathscr{L}(\mathbf{x})} \leqq C \int d \mathbf{x}\left|H^{\mathbf{m r}}\left(x_{j}\right)\right| e^{2 \mathscr{L}(\mathbf{x})} .
$$

The change $\mathscr{L}(\mathbf{x}) \rightarrow 2 \mathscr{L}(\mathbf{x})$ can be easily accommodated due to the scaling of distances by $L$ in the RG transformation, see e.g. (114). Thus

$$
\left\|\tilde{H}_{n-1}^{4}\right\| \leqq L^{-1}\left\|\tilde{H}_{n}^{4}\right\|+\mathcal{O}\left(g_{n}^{3}\right)
$$

and (92) for $\tilde{H}_{n-1}^{4}$ follows. $H_{n-1}^{2}$ is similar (but easier). The induction is completed.

\section{The Continuum Limit}

The preceding analysis has shown that the effective actions stay bounded as the cutoff is removed. Now we show that they actually converge as $\Lambda \rightarrow \infty$,

$$
H_{\tilde{\Lambda}}^{\Lambda} \equiv R_{\Lambda / \tilde{\Lambda}} H^{\Lambda} \underset{\Lambda \rightarrow \infty}{\longrightarrow} H_{\tilde{\Lambda}} .
$$

As before, we take $\Lambda=L^{n_{0}}, \tilde{\Lambda}=L^{n}$ and denote $H_{\tilde{\Lambda}}^{\Lambda} \equiv H_{n}^{n_{0}}$, often dropping superscript $n_{0}$ if it does not lead to a confusion.

We shall study the variation of $H_{n}^{n_{0}}$ under the change of the cutoff:

$$
\delta H_{n}^{n_{0}} \equiv H_{n}^{n_{0}+1}-H_{n}^{n_{0}} .
$$

With $n$ fixed, we get for the UV limit

$$
H_{n}^{\infty}=H_{n}^{n}+\sum_{n_{0}=n}^{\infty} \delta H_{n}^{n_{0}} .
$$

Thus convergence of (135) has to be proven. It is easy to derive a recursion for $\delta H_{n}^{n_{0}}$ in $n$,

$$
\delta H_{n-1}^{n_{0}}=-\log \left(\left\langle e^{-H_{n}^{I} n_{0}+1}\right\rangle_{\Gamma} /\left\langle e^{-H_{n}^{I} n_{0}}\right\rangle_{\Gamma}\right)=-\log \int d v_{n}^{n_{0}} e^{-\delta H_{n}^{n_{0}}}
$$

where

$$
d v_{n}^{n_{0}}=e^{-H_{n}^{I} n_{0}\left(\tilde{\psi}_{L}+\cdot\right)} d \mu_{\Gamma}(\cdot) / \int e^{-H_{n}^{I} n_{0}\left(\tilde{\psi}_{L}+\tilde{\zeta}\right)} d \mu_{\Gamma}(\tilde{\zeta})
$$

Hence

$$
\delta H_{n-1}^{n_{0}}=\sum_{k=1}^{\infty} \sum_{\ell=0}^{\infty} \frac{(-)^{k+\ell+1}}{k ! \ell !}\left\langle\left(\delta H_{n}^{n_{0}}\right)^{k}\left(H_{n}^{I n_{0}}\right)^{\ell}\right\rangle_{\Gamma}^{T}
$$


We write again

$$
\delta H_{n}^{n_{0}}=\sum_{m \leqq 8} \sum_{\mathbf{A}} \int d \mathbf{x} \delta H_{n}^{n_{0} m}(\mathbf{x}, \mathbf{A}): \Psi(\mathbf{x}, \mathbf{A}):+\sum_{m>8} \sum_{\mathbf{A}} \int d \mathbf{x} \delta H_{n}^{n_{0} m}(\mathbf{x}, \mathbf{A}) \Psi(\mathbf{x}, \mathbf{A}),
$$

and compute first $\delta H_{n_{0}-1}^{n_{0}}$. From (21), (68), (96), and (97), as in (100), we obtain

$$
\delta g_{n_{0}}^{n_{0}} \equiv g_{n_{0}}^{n_{0}+1}-g^{n_{0}}=\left(B_{*}-B_{0}\right)\left(g^{n_{0}}\right)^{3}+\mathcal{O}\left(\left(g^{n_{0}}\right)^{3+2 \varepsilon}\right),
$$

say. Similarly, (78) gives

$$
\delta z_{n_{0}}^{n_{0}}=\mathcal{O}\left(\left(g^{n_{0}}\right)^{3}\right) .
$$

The rest of the terms are as in $H_{n_{0}}^{n_{0}+1}$, cf. (69) to (71) and (74) to (76).

Let us first see what type of iteration for $\delta g_{n}^{n_{0}}$ may be expected. Formally,

$$
\tilde{\Lambda} \frac{d}{d \tilde{\Lambda}} \delta g_{\tilde{\Lambda}}=2 \beta_{2} g_{\tilde{\Lambda}} \delta g_{\tilde{\Lambda}}
$$

where we have ignored all higher order terms. Taking

$$
g_{\Lambda}=g_{\mu}\left(1-\beta_{2} g_{\mu} \log \Lambda\right)^{-1}
$$

we obtain by solving (142):

$$
\delta g_{\tilde{\Lambda}}=\delta g_{\Lambda} g_{\tilde{\Lambda}}^{2} / g_{\Lambda}^{2}
$$

Thus the initial $\delta g_{\Lambda}$ has to be smaller than, say, $\mathcal{O}\left(g_{\Lambda}^{3+\varepsilon}\right)$ to produce $\left|\delta g_{\tilde{\Lambda}}\right|$ $\leqq \mathcal{O}\left(g_{\Lambda}^{1+\varepsilon} g_{\tilde{\Lambda}}^{2}\right)$, which is integrable over $\log \Lambda>\log \tilde{\Lambda}$, and thus would yield convergence in (135). The initial value $\delta g_{A}$ depends on how accurately we choose the bare coupling. Our choice (21), dictated by the solution of the flow equation to the third order, gives in principle $\delta g_{A}=\mathcal{O}\left(g_{A}^{4}\right)$, reflected by (140) [the first term on the right-hand side of (140) reflects the deviation of the initial effective Hamiltonians from the renormalized trajectory]. Notice that taking $g^{\Lambda}$ given by the leading logarithms approximation would produce $\delta g_{\Lambda}=\mathcal{O}\left(g_{\Lambda}^{3}\right)$, which would be insufficient.

Let us pass to the exact bounds now. By induction we shall prove the following estimates: $\left(\lambda \equiv L^{n_{0}-n}, g \equiv g_{n_{0}}\right)$

$$
\begin{gathered}
\left|\delta z_{n}\right| \leqq \alpha_{1} g^{1+\varepsilon} g_{n}^{2-\varepsilon}, \\
\left\|\delta H_{n}^{2}\right\| \leqq \alpha_{2} \lambda^{1 / 2} g_{n}^{2}+\alpha_{3} g^{1+\varepsilon} g_{n}^{3-\varepsilon}, \\
\left|\delta g_{n}-\left(B^{*}-B_{n_{0}-n}\right) g_{n}^{3}\right| \leqq \alpha_{4} g^{1+\varepsilon} g_{n}^{2}, \\
\delta H_{n}^{4}(\mathbf{x})=g_{n}^{2} \lambda L_{1}\left(\lambda x_{2}, \lambda x_{4}\right)+z_{n} g_{n} \lambda K_{1}\left(\lambda x_{1}, \lambda x_{4}\right)+\delta \tilde{H}_{n}^{4}(\mathbf{x}), \\
\left\|\delta \tilde{H}_{n}^{4}\right\| \leqq \alpha_{5} \lambda^{1 / 2} g_{n}^{3}+\alpha_{6} g^{1+\varepsilon} g_{n}^{3-\varepsilon}, \\
\delta H_{n}^{6}(\mathbf{x})=g_{n}^{2} \lambda M_{1}\left(\lambda x_{3}, \lambda x_{6}\right)+\delta \tilde{H}_{n}^{6}(\mathbf{x}), \\
\left\|\delta \tilde{H}_{n}^{6}\right\| \leqq \alpha_{7} \lambda^{1 / 2} g_{n}^{3+\varepsilon(m-8)}+\alpha_{8} g^{1+\varepsilon} g_{n}^{3}, \\
\left\|\delta H_{n}^{m}\right\| \leqq \alpha_{9}^{m} \lambda^{1 / 2} g_{n}^{3+\varepsilon(m-8)}+\alpha_{10}^{m} g^{1+\varepsilon} g_{n}^{4+\varepsilon(m-8)}
\end{gathered}
$$

Equations (144) to (150) are clearly satisfied for $n=n_{0}$. Since the perturbation expansion (138) converges as in Sect. 6, we only need to collect the leading terms in order to show that the bounds iterate. 
I. For $\delta z_{n}$ we easily get

$$
\left|\delta z_{n-1}-\delta z_{n}\right| \leqq \lambda^{1 / 2} \mathcal{O}\left(g_{n}^{3}\right)+\mathcal{O}\left(g^{1+\varepsilon} g_{n}^{3}\right),
$$

since the leading contribution comes from $\gamma_{2} g_{n} \delta g_{n} \log L$. Hence

$$
\left|\delta z_{n-1}\right| \leqq\left(\alpha_{1}+\mathcal{O}\left(\lambda^{1 / 4}\right)+\mathcal{O}\left(g_{n}^{1+\varepsilon}\right)\right) g^{1+\varepsilon}+g_{n-1}^{2-\varepsilon},
$$

as $\lambda^{1 / 4} g_{n}^{1+\varepsilon} \leqq \mathcal{O}\left(g^{1+\varepsilon}\right)$. The expression in the parenthesis in (152) is summable over $n$ so that (144) follows.

II. $\delta H_{n}^{2}$ contracts in the linear approximation [this allows us to add up one $L^{-1 / 2}$ factor in the first term of the bound (145)]. New leading contributions are $\mathcal{O}\left(\delta z_{n} z_{n}\right)$. This gives (145).

III. $\delta g_{n}$ is the most subtle. We have

$$
\begin{aligned}
\delta g_{n-1}= & \delta g_{n}-2 \beta_{2} g_{n} \delta g_{n} \log L+\mathcal{O}\left(g_{n}^{2}\right) \delta g_{n}+\delta b_{n_{0}-n} g_{n}^{3} \\
& +\delta c_{n_{0}-n} z_{n} g_{n}^{2}+\mathcal{O}\left(\lambda^{1 / 2} g_{n}^{3+2 \varepsilon}\right)+\mathcal{O}\left(g^{1+\varepsilon} g_{n}^{4-\varepsilon}\right)
\end{aligned}
$$

Note that the main terms of (153) can be obtained formally by taking the variation of (94), which explains their origin. $\mathcal{O}\left(\lambda^{1 / 2} g_{n}^{3+2 \varepsilon}\right)$ has its main contribution from the term linear in $\delta H_{n}^{10}$ and $\mathcal{O}\left(g^{1+\varepsilon} g_{n}^{4-\varepsilon}\right)$ from $\mathcal{O}\left(\delta z_{n} g_{n}^{2}\right)$ and $\mathcal{O}\left(\delta \tilde{H}^{4} g^{n}\right)$. Now, by (80), (95), and (96),

$$
\delta b_{n_{0}-n} g_{n}^{3}+\delta c_{n_{0}-n_{n}} z_{n} g_{n}^{2}=-\delta B_{n_{0}-n} g_{n}^{3}+\mathcal{O}\left(\lambda g_{n}^{4}\right) .
$$

Using (146) and (154), we obtain from (153)

$$
\begin{aligned}
\left|\delta g_{n-1}-\left(B_{*}-B_{n_{0}-n+1}\right) g_{n-1}^{3}\right| \leqq & \alpha_{4}\left(1-2 \beta_{2} g_{n} \log L\right) g^{1+\varepsilon} g_{n}^{2} \\
& +\mathcal{O}\left(\lambda^{1 / 2} g_{n}^{3+2 \varepsilon}\right)+\mathcal{O}\left(g^{1+\varepsilon} g_{n}^{4-\varepsilon}\right) .
\end{aligned}
$$

But by (79),

$$
\left(1-2 \beta_{2} g_{n} \log L\right) g_{n}^{2}=g_{n-1}^{2}+\mathcal{O}\left(g_{n}^{4}\right)
$$

Thus

$$
(155) \leqq\left(\alpha_{4}+\mathcal{O}\left(\lambda^{1 / 4}\right)+\mathcal{O}\left(g_{n}^{2-\varepsilon}\right)\right) g^{1+\varepsilon} g_{n-1}^{2}
$$

Since the $\mathcal{O}\left(L^{1 / 4\left(n-n_{0}\right)}\right)+\mathcal{O}\left(g_{n}^{2-\varepsilon}\right)$ are summable in $n$, this establishes (146).

IV. From $\delta H_{n}^{4}$ we single out the $\mathcal{O}\left(g_{n}^{2}\right)$ and $\mathcal{O}\left(z_{n} g_{n}\right)$ parts, which easily iterate. The rest is driven by a contracting $\mathcal{O}\left(g_{n}^{3}\right)$ term and the $\mathcal{O}\left(\delta z_{n} g_{n}\right)$ contributions.

V. $\delta H_{n}^{m}$ for $m \geqq 6$, besides the contracting part, gains each time contributions, having among the leading terms, for example, the ones coming from the graphs of Fig. 3 with one $\delta g_{n}$ vertex. From the contracting part of $\delta H_{n}^{6}$ we single out the $\mathcal{O}\left(g_{n}^{2}\right)$ term.

This establishes the bounds (144) to (150), which in turn imply convergence of (135) and existence of the UV limit $\Lambda \rightarrow \infty$. Since all our bounds are volume independent, existence of the infinite volume $l \rightarrow \infty$ limit is an easy exercise in the application of the Dominated Convergence Theorem. 


\section{The Chirally Invariant Model}

The analysis of the chirally invariant model (12) is almost identical to the one of the $(\bar{\psi} \psi)^{2}$ theory. The only difference is that there are two couplings now $(N>1)$. In the second order of the perturbation expansion, the flow of the couplings is [3]

$$
\begin{aligned}
& g_{n-1}=g_{n}+\frac{2 N}{\pi} g_{n}^{2} \log L+\mathcal{O}\left(g^{3}\right), \\
& g_{v n-1}=g_{v n}-\frac{2}{\pi} g_{n}^{2} \log L+\mathcal{O}\left(g^{3}\right) .
\end{aligned}
$$

Thus the vector-vector coupling constant flows with $g_{n}$ similarly as the wave function renormalization $z_{n}: g_{v n}=-\frac{1}{N} g_{n}+O\left(g_{n}^{2}\right)$.

For $N=1$ the two couplings are no more independent as $\left(\bar{\psi} \gamma_{\mu} \psi\right)^{2}$ $=(\bar{\psi} \psi)^{2}-\left(\bar{\psi} \gamma_{5} \psi\right)^{2}$ in this case by a Fierz identity. The overall increment of $g+g_{v}$ given in the second order by (157) and (158) is zero. Indeed, the complete $\beta$ function vanishes by the exact solution of this Thirring model [13].

\section{The Mass Term}

We can analyze the change of the effective interactions due to the presence of the mass term as a perturbation $\Delta H_{n}^{n_{0}} \equiv H_{n}^{n_{0}}-H_{n}^{n_{0}}$, where $H_{n}^{n_{0}}$ is the effective Hamiltonian with the mass term. The flow of $\Delta H_{n}^{n_{0}}$ may be studied recursively in convergent perturbation theory since [see (137)]

$$
\Delta H_{n-1}^{n_{0}}=-\log \int e^{-\Delta H_{n}^{n_{0}}} d v_{n}^{n_{0}}=\sum_{k=1}^{\infty} \sum_{\ell=0}^{\infty} \frac{(-)^{k+\ell+1}}{k ! \ell !}\left\langle\left(\Delta H_{n}^{n_{0}}\right)^{k}\left(H_{n}^{I n_{0}}\right)^{\ell}\right\rangle_{\Gamma}^{T} .
$$

Denoting the coefficient in $\Delta H_{n}^{n_{0}}$ at $\bar{\psi} \psi$ by $r_{n}$ (dimensionless mass!), we get in the lowest orders [for the $(\bar{\psi} \psi)^{2}$ model]

$$
r_{n-1}=L r_{n}\left(1-c g_{n} \log L\right)+\mathcal{O}\left(r_{n} g_{n}^{2}, r_{n}^{2}\right) .
$$

Solving this recursion yields

$$
r_{n} \approx L^{n_{0}-n}\left(g_{n} / g_{n_{0}}\right)^{+c / \beta_{2}} r_{n_{0}} .
$$

This explains our choice (27) of the bare mass $\left(v=-\frac{c}{\beta_{2}}, m^{\Lambda}=\frac{1}{\mu} r^{\Lambda}\right)$. The rigorous analysis of the recursion (159), as well as of the other one for $\delta \Delta H_{n}^{n_{0}}$ $=\Delta H_{n}^{n_{0}+1}-\Delta H_{n}^{n_{0}}$ used to establish the existence of the ultraviolet limit in the presence of the mass term is left to the reader as a standard exercise in the application of the method developed in the present paper.

\section{Appendix 1}

In this appendix, we prove a useful estimate for the free, ultraviolet and infrared cutoff Green functions. This is a little more subtle with the momentum cutoff than 
on the lattice, since $\psi_{\alpha}(x) \psi_{\alpha}(x+\varepsilon) \neq 0$ for each $\varepsilon \neq 0$. Basically, the idea still is that localized Green functions with many fields are heavily suppressed, since effectively we have finite number of degrees of freedom per unit volume. Thus consider the following Green function,

$$
\Gamma_{n}\left(\Delta_{1}, \Delta_{2}\right) \equiv\left\langle\prod_{i=1}^{n} \zeta_{\alpha_{i}}\left(x_{i}\right) \bar{\zeta}_{\beta_{i}}\left(y_{i}\right)\right\rangle_{\Gamma},
$$

where all $x_{i} \in \Delta_{1}$ and all $y_{i} \in \Delta_{2}, \Delta_{1}, \Delta_{2}$ being unit cubes, and $\alpha_{i}$ and $\beta_{i}$ refer to spin and flavor indices. We show that

$$
\left|\Gamma_{n}\right| \leqq \frac{1}{(n !)^{2 \alpha}} C(L, \alpha)^{n} e^{-4 n \operatorname{dist}\left(\Delta_{1}, \Delta_{2}\right)}
$$

for any $\alpha$ : local powers of $\tilde{\zeta}$ are suppressed!

To prove (A.2), first note that

$$
\Gamma_{n}=\operatorname{det}_{i, j} \Gamma_{\alpha_{i} \beta_{j}}\left(x_{i}-x_{j}\right) \equiv \operatorname{det} \Gamma_{\{i\}\{j\}} .
$$

We divide the analysis into two cases:

$$
\begin{aligned}
& \operatorname{dist}\left(\Delta_{1}, \Delta_{2}\right) \leqq a \log n, \\
& \operatorname{dist}\left(\Delta_{1}, \Delta_{2}\right)>a \log n .
\end{aligned}
$$

$a$ will be explained below. We shall work with infinite volume covariance $\Gamma$. The proof easily extends to the case of finite cutoff $\ell$ giving $\ell$ independent bounds. (A). Write $\Gamma$ as

$$
\Gamma(x-y)=\Gamma^{0}(x-y)+\Gamma^{c}(x-y),
$$

where $\Gamma^{0}$ is $\Gamma$ with periodic boundary conditions in the box of side $R=2 a \cdot \log n$ centered at zero (take the origin between $\Delta_{1}$ and $\Delta_{2}$ ). Thus

$$
\Gamma^{0}(x-y)=\sum_{a \in \mathbb{Z}^{2}} \Gamma(x-y+a R)=\sum_{p \in \frac{2 \pi}{R} \mathbb{Z}^{2}} R^{-2} e^{i p(x-y)} \hat{\Gamma}(p),
$$

where

$$
\hat{\Gamma}(p)=\frac{\not p}{p^{2}}\left(e^{-p^{2}}-e^{-L^{2} p^{2}}\right) .
$$

Since $\Gamma(x)$ decays exponentially, we get

$$
\left|\Gamma^{c}(x-y)\right| \leqq C(L) e^{-3 R}
$$

say. Now

$$
\operatorname{det} \Gamma_{\{i\}\{j\}}=\sum_{I J}(-)^{\#} \operatorname{det} \Gamma_{I J}^{0} \operatorname{det} \Gamma_{I^{c} J^{c}}^{c},
$$

where $I, J \subset\{1, \ldots, n\},|I|=|J| \equiv m$. Inequality (A.7) gives

$$
\left|\operatorname{det} \Gamma_{I^{c} J^{c}}^{c}\right| \leqq(n-m) ! C(L)^{n-m} e^{-3 R(n-m)} .
$$

As for $\operatorname{det} \Gamma_{I J}^{0}$, note that

$$
\left|\operatorname{det} \Gamma_{I J}^{0}\right|=\left|\left\langle\prod_{i \in I} \zeta_{\alpha_{i}}\left(x_{i}\right) \prod_{j \in J} \bar{\zeta}_{\beta_{j}}\left(y_{j}\right)\right\rangle_{\Gamma^{0}}\right|,
$$


where

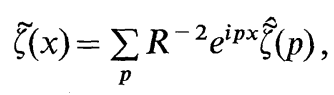

and $\zeta_{\alpha}(p)^{2}=\bar{\zeta}_{\alpha}(p)^{2}=0$

$$
\langle\zeta(p) \bar{\zeta}(q)\rangle_{\Gamma^{0}}=R^{2} \hat{\Gamma}(p) \delta_{p,-q} .
$$

Thus (A.10) is bounded by

$$
m ! \sum_{p_{i} \in \frac{2 \pi}{R} \mathbb{Z}^{2}}^{\prime} R^{-2 m} \prod_{i=1}^{m} \hat{\Gamma}\left(p_{i}\right) \leqq m !(C(L) / R)^{2 m} \sum_{p_{i} \in \mathbb{Z}^{2}}^{\prime} e^{-\frac{\Sigma}{i}\left(\frac{2 \pi}{R}\right)^{2} p_{t}^{2}},
$$

where at most $2 N$ of the $p_{i}$ 's may coincide. This implies that $p_{i}$ 's must at worst fill a ball in $\mathbb{Z}^{2}$ of radius $\geqq \mathrm{Cm}^{1 / 2}$, and hence

$$
\sum_{i} p_{i}^{2} \geqq C m^{2} .
$$

Using (A.14) in (A.13), we obtain

$$
\left|\operatorname{det} \Gamma_{I J}^{0}\right| \leqq m !\left(C(L) R^{-1}\right)^{2 m} \int_{p^{2} \geqq C m^{2}} e^{-C p^{2} / R^{2}} d^{2 m} p \leqq m ! C(L)^{m} \exp \left[-C m^{2} / R^{2}\right] .
$$

Equations (A.8), (A.9), and (A.15) give

$$
\left|\operatorname{det} \Gamma_{\{i\}\{j\}}\right| \leqq n ! C(L)^{n} \exp \left[-C m^{2} R^{-2}-3 R(n-m)\right] .
$$

But

$$
\max \left(3 R m-\frac{C m^{2}}{R^{2}}\right) \leqq C R^{4} \leqq C a^{4}(\log n)^{4}
$$

Hence

$$
\text { (A.16) } \leqq n ! C(L)^{n} e^{-n a \log n} e^{-2 n R} \leqq \frac{C(a, L)^{n}}{(n !)^{a-1}} e^{-4 n \operatorname{dist}\left(\Delta_{1}, \Delta_{2}\right)},
$$

which yields (A.2) for $a=1+2 \alpha$.

(B). By brute force estimation,

$$
\left|\operatorname{det} \Gamma_{\{i\}\{j\}}\right| \leqq n ! C(L)^{n} e^{-5 n \operatorname{dist}\left(\Delta_{1}, \Delta_{2}\right)} \leqq n ! C(L)^{n} e^{-n a \log n} e^{-4 n \operatorname{dist}\left(\Delta_{1}, \Delta_{2}\right)},
$$

and the claim follows.

Obviously (A.2) still holds if some of the fields in (A.1) are differentiated, as derivatives of $\Gamma(x-y)$ can be accommodated in the bounds we have used.

\section{Appendix 2}

Here we shall derive a convenient representation for (partially) truncated correlations. Let us start with the expectation

$$
E \equiv\left\langle\prod_{k=1}^{N}\left(\prod_{i \in I_{k}} \zeta_{\alpha_{i}}\left(x_{i}\right) \prod_{j \in J_{k}} \bar{\zeta}_{\beta_{j}}\left(y_{j}\right)\right)\right\rangle_{\Gamma}
$$


Again we may also admit derivatives of $\widetilde{\zeta}$ 's (of bounded order). The sets of indices $I_{k}$ and $J_{k}$ are assumed to be disjoint. For $\Delta$ being a unit lattice square, let

$$
I_{k \Delta}=\left\{i \in I_{k}: x_{i} \in \Delta\right\}, \quad I_{\Delta}=\bigcup I_{k \Delta},
$$

and similarly for $J$. Let

$$
X_{k}=\left(\bigcup_{I_{k} \Delta \cup J_{k \Delta} \neq \emptyset} \Delta\right) \cap \mathbb{Z}^{2}, \quad X=\bigcup_{k} X_{k} .
$$

Exhibiting the "geography" of the pairings in the expectation (A.20), we may write

$$
E=\sum_{\left\{I_{\Delta}^{\Delta}, J_{\Delta}^{\prime}\right\}}(-)^{\#} \prod_{\left(\Delta, \Delta^{\prime}\right)} \operatorname{det} \Gamma_{I_{\Delta}^{\prime} J \Delta^{\prime},}
$$

where $\left\{I_{\Delta}^{\Delta^{\prime}}\right\}_{\Delta^{\prime}}$ forms a partition of $I_{\Delta}$ (possibly with empty sets), $\left\{J_{\Delta^{\prime}}^{\Delta}\right\}_{\Delta}$ of $J_{\Delta^{\prime}}$, $\left|I_{\Delta^{\prime}}^{A}\right|=\left|J_{\Delta}^{A^{\prime}}\right|$ and \# gives the parity of the permutation which brings the original product of fields in (A.20) into the one implied in (A.23).

Now associate to a term of (A.23) a graph $\mathscr{G}$ of lines joining pairs of different points $x, x^{\prime} \in X$, centers of unit squares $\Delta, \Delta^{\prime}$, in the following way: $x, x^{\prime}$ are joined by a line if

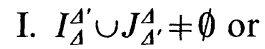

II. for some $k, I_{k \Delta} \cup J_{k \Delta} \neq \emptyset \neq I_{k \Delta^{\prime}} \cup J_{k \Delta^{\prime}}$.

Thus $X_{k}$ 's are by definition connected, the other lines of $\mathscr{G}$ being provided by the propagators $\Gamma$ of (A.23). The decomposition of $\mathscr{G}$ into connected components induces a partition $\left\{X_{a}\right\}$ of the "support" $X$ of $E$. Let

$$
K_{a}=\left\{k: X_{k} \subset X_{a}\right\} .
$$

Notice that $\left\{K_{a}\right\}$ is a partition of $\{1, \ldots, N\}$ (without empty sets). Given a set $K \subset\{1, \ldots, N\}$, let us define

$$
\varrho(K)=\sum_{\left\{\Delta_{\Delta}^{\prime}, J_{\Delta}^{\Delta},\right\}}^{\prime}(-)^{\#} \prod_{\left(\Delta, \Delta^{\prime}\right)} \operatorname{det} \Gamma_{I_{\Delta}^{\prime} J_{\Delta}^{\prime},},
$$

where the right-hand side is as (A.23) except that we consider an expectation like in (A.20) but with $k$ running only through $K$ and the summation in (A.25) is restricted to terms producing connected graphs $\mathscr{G}$ on the points of $X_{K} \equiv \bigcup_{k \in K} X_{k}$. Notice that

$$
E=\sum_{\left\{K_{a}\right\}}^{\prime}(-)^{\#} \prod_{a} \varrho\left(K_{a}\right),
$$

where the sum runs over the partitions $\left\{K_{a}\right\}$ of $\{1, \ldots, N\}$ such that $\left\{X_{K_{a}}\right\}$ are disjoint and \# is a parity depending only on the partition. We shall rewrite (A.26) as an unconstrained sum over all the partitions

$$
E=\sum_{\left\{K_{a}\right\}}(-)^{\#} \prod_{\mathscr{L}} U(\mathscr{L}) \prod_{a} \varrho\left(K_{a}\right),
$$

where $\mathscr{L}$ runs over the unordered pairs of different sets of the partition $\left\{K_{a}\right\}$ with

$$
U\left(K_{a}, K_{a^{\prime}}\right)= \begin{cases}1 & \text { if } X_{K_{a}} \cap X_{K_{\alpha^{\prime}}}=\emptyset, \\ 0 & \text { otherwise. }\end{cases}
$$

Writing $U \equiv(1+A)$ and expanding $\prod_{\mathscr{L}}(1+A(\mathscr{L}))$, we obtain from (A.27),

$$
E=\sum_{\left\{K_{a}\right\}}(-)^{\#} \sum_{\Gamma} \prod_{\mathscr{L} \in \Gamma} A(\mathscr{L}) \prod_{a} \varrho\left(K_{a}\right),
$$


where $\Gamma$ runs through the collections of "lines" $\mathscr{L}$ (Mayer graphs on vertices $K_{a}$ ). Grouping all the vertices $K_{a}$ of the connected components $\Gamma_{c}$ of $\Gamma$ together, we obtain a partition $\left\{H_{b}\right\}$ of $\{1, \ldots, N\}$. Equation (A.29) becomes

$$
E=\sum_{\left\{H_{b}\right\}}(-)^{\#} \prod_{b}\left(\sum_{\substack{\left\{K_{a}\right\} \\ \text { part. of } H_{b}}}(-)^{\#} \sum_{\Gamma_{c}} \prod_{\mathscr{L} \in \Gamma_{c}} A(\mathscr{L}) \prod_{a} \varrho\left(K_{a}\right)\right),
$$

where the first \# is the parity of $\left\{H_{b}\right\}$ and the second one is the parity of $\left\{K_{a}\right\}$ relative to $H_{b}$. On the other hand, the expectation (A.20) can be expressed by truncated expectations (with truncations between $N$ groups of variables) as

$$
E=\sum_{\left\{H_{b}\right\}}(-)^{\#} \prod_{b}\left\langle\prod_{k \in H_{b}}\left(\prod_{i \in I_{k}} \zeta_{\alpha_{i}}\left(x_{i}\right) \prod_{j \in J_{k}} \bar{\zeta}_{\beta_{j}}\left(y_{j}\right)\right)\right)_{\Gamma}^{T},
$$

which in fact defines the truncated expectations. From (A.30) and (A.31), we read off (the parities agree)

$$
E^{T} \equiv\left\langle\prod_{k=1}^{N}\left(\prod_{i \in I_{k}} \zeta_{a_{i}}\left(x_{i}\right) \prod_{j \in J_{k}} \bar{\zeta}_{\beta_{j}}\left(y_{j}\right)\right)\right\rangle_{\Gamma}^{T}=\sum_{\left\{K_{a}\right\}}(-)^{\#} \sum_{\Gamma_{c}} \prod_{\mathscr{L} \in \Gamma_{c}} A(\mathscr{L}) \prod_{a} \varrho\left(K_{a}\right) .
$$

This is the formula which serves as a basis for the estimation of the truncated expectations which we do in

\section{Appendix 3}

Let us start with showing that

$$
|\varrho(K)| \leqq C^{\left|I_{K}\right|}\left(\prod_{\Delta}\left(\left|I_{K \Delta}\right| !\left|J_{K \Delta}\right| !\right)^{-\alpha} e^{-2 \mathscr{L}\left(\left\{X_{k}\right\} k \in K\right)},\right.
$$

where $I_{K}=\bigcup_{k \in K} I_{k}, I_{K \Delta}=\bigcup_{k \in K} I_{k \Delta}$, and similarly for $J$, and $\mathscr{L}\left(\left\{X_{k}\right\}_{k \in K}\right)$ is the length of the shortest graph on the points of $X_{K}$ and possibly other points which become connected when $X_{k}$ 's are shrunk to points.

To show (A.33), use first (A.2) and (A.25) to get

$$
\begin{aligned}
|\varrho(K)| \leqq & C^{\left|I_{K}\right|} e^{-2 \mathscr{L}\left(\left\{X_{k}\right\}\right)} \sum_{\left\{\Delta_{\Delta}^{\Delta^{\prime}}, J_{\left.\Delta^{\prime},\right\}}\right.} \prod_{\left(\Delta, \Delta^{\prime}\right)}\left(\left|I_{\Delta}^{\Delta^{\prime}}\right| !\left|J_{\Delta^{\prime}}^{\Delta}\right| !\right)^{-\alpha} \\
& \cdot \exp \left[-\left(\left|I_{\Delta}^{\Delta^{\prime}}\right|+\left|J_{\Delta^{\prime}}^{\Delta}\right|\right) \operatorname{dist}\left(\Delta, \Delta^{\prime}\right)\right] .
\end{aligned}
$$

Consider the sum over $\left\{I_{\Delta}^{\Delta^{\prime}}\right\}, \Delta$ fixed. Order $\Delta^{\prime}$ for which $J_{\Delta^{\prime}} \neq \emptyset$ as $\Delta_{1}^{\prime}, \Delta_{2}^{\prime}, \ldots$, so that $d\left(\Delta, \Delta_{1}^{\prime}\right) \leqq d\left(\Delta, \Delta_{2}^{\prime}\right) \leqq \ldots$. Denote $\left|I_{\Delta}^{\Delta_{j}^{\prime}}\right|=p_{j} \cdot \sum p_{j}=\left|I_{\Delta}\right|$. Thus

$$
\begin{aligned}
\sum_{\left\{\Delta_{\Delta}^{\Delta^{\prime}}\right\}}^{\prime} \prod_{\Delta^{\prime}}\left(\left|I_{\Delta^{\prime}}^{\Delta}\right| !\right)^{-\alpha} e^{-\left|I_{\Delta}^{\Delta}\right| \operatorname{dist}\left(\Delta, \Delta^{\prime}\right)} & =\sum_{\left(p_{j}\right)}^{\prime} \frac{\left|I_{\Delta}\right| !}{\left(\prod p_{j} !\right)^{\alpha+1}} e^{-\Sigma p_{j} d\left(\Delta, \Delta_{j}^{\prime}\right)} \\
& \leqq \sum_{\left(p_{j}\right)} \frac{\left|I_{\Delta}\right| !}{\left(\prod p_{j} !\right)^{\alpha+1}} e^{-\sum_{j} p_{j} j^{1 / 2} / C}
\end{aligned}
$$

as $\operatorname{dist}\left(\Delta, \Delta_{j}^{\prime}\right) \geqq j^{1 / 2} / C$ for some $C$. Find

$$
\max _{\Sigma p_{j}=\left|I_{\Delta}\right|} \prod_{j} p_{j}^{-(\alpha+1) p_{j}} \exp \left[-\frac{1}{2 C} p_{j} j^{1 / 2}\right] .
$$

It is easily seen that the maximum is attained at the solution of the extremal equations

$$
(\alpha+1) \log p_{j}+\alpha+1+\frac{1}{2 C} j^{1 / 2}-\lambda=0 .
$$


Thus

$$
(\mathrm{A} .36) \leqq \prod_{j} e^{p_{J}(\alpha+1-\lambda)}=e^{\left|I_{\Delta}\right|(\alpha+1-\lambda)} .
$$

To eliminate $\lambda$, compute from (A.37)

$$
p_{j}=\exp \left[\frac{\lambda}{\alpha+1}-1-\frac{1}{2 C(\alpha+1)} j^{1 / 2}\right]
$$

so that

$$
\left|I_{\Delta}\right|=e^{\frac{\lambda}{\alpha+1}-1} \sum_{j} e^{-\frac{1}{2 C(\alpha+1)} j^{1 / 2}} \leqq C e^{\frac{\lambda}{\alpha+1}-1} .
$$

From (A.38) and (A.40), we obtain

$$
(\mathrm{A} .36) \leqq C^{\left|I_{\Delta}\right|}\left(\left|I_{\Delta}\right| !\right)^{-\alpha-1} .
$$

Expressions (A.35), (A.36), and (A.41) imply that

$$
(\mathrm{A} .35) \leqq C^{\left|I_{\Delta}\right|}\left(\left|I_{\Delta}\right| !\right)^{-\alpha} \prod_{j}\left(\sum_{p} e^{-\frac{1}{2 C} p_{j}^{1 / 2}}\right) \leqq C^{\left|I_{\Delta}\right|} /\left(\left|I_{\Delta}\right| !\right)^{\alpha} .
$$

Bounding similarly the sum over $\left\{J_{\Delta^{\prime}}^{4}\right\}$ with $\Delta^{\prime}$ fixed in (A.34), we obtain (A.33).

Let us pass to the estimation of (A.32). This is a typical Ursel function expression and we shall closely follow the standard procedure, see [14 and 15, Theorem 3.6 and pp. 47-48]. First notice that (A.32) may be rewritten as

$$
E^{T}=\sum_{\left(K_{a}\right)}^{\prime} \frac{(-)^{\#}}{A !} \prod_{a=1}^{A} \varrho\left(K_{a}\right) a(G)
$$

where the sum over ordered partitions is restricted by demanding that the graph $G$ on vertices $K_{a}$ formed by all the lines $\mathscr{L}$ with $A(\mathscr{L})=-1$ (i.e. by the lines between the vertices with $X_{K_{a}}$ intersecting) be connected. $a(G)=\sum_{\Gamma_{c} \subset G}(-)^{\left|\Gamma_{c}\right|}$. The crucial
combinatorial estimate is

$$
|a(G)| \leqq|T(G)|
$$

where $T(G)=\sum_{\text {trees } \Gamma_{\mathrm{c}} \subset G} 1$, see e.g. Theorem 3.6 of [15]. Thus

$$
\left|E^{T}\right| \leqq \sum_{\left(K_{a}\right)}^{\prime} \frac{1}{A !} \sum_{\text {trees } \Gamma_{c} \subset G} \prod_{a=1}^{A}\left|\varrho\left(K_{a}\right)\right| .
$$

With the use of (A.33), this yields

$$
\begin{aligned}
\left|E_{T}\right| \leqq & \prod_{k=1}^{N} C^{\left|I_{k}\right|}\left(\prod_{\Delta}\left|I_{k \Delta}\right| !\left|J_{k \Delta}\right| !\right)^{-\alpha} \sum_{\left(K_{a}\right)}^{\prime} \frac{1}{A !} \sum_{\Gamma_{c}} \prod_{a} e^{-2 \mathscr{L}\left(\left\{X_{k}\right\}_{k \in K_{a}}\right)} \\
\leqq & \prod^{\left|I_{k}\right|} e^{1 / 2 \mathscr{L}\left(X_{k}\right)}\left(\prod\left|I_{k \Delta}\right| !\left|J_{k \Delta}\right| !\right)^{-\alpha} e^{\left.-\mathscr{L}\left(\left\{X_{k}\right\}\right\}_{k=1}^{N}\right)} \\
& \cdot \sum_{\left(K_{a}\right)}^{\prime} \frac{1}{A !} \sum_{\Gamma_{c}} \prod_{a} \exp \left[-\frac{1}{4} \mathscr{L}\left(X_{K_{a}}\right)-\frac{1}{4} \sum_{k \in K_{a}} \mathscr{L}\left(X_{k}\right)\right] .
\end{aligned}
$$

In (A.46) we have extracted $e^{\left.-\mathscr{L}\left(\left\{X_{k}\right\}\right\}_{k}^{N}=1\right)}$, which is bigger or equal to $\prod_{a} e^{-\mathscr{L}\left(\left\{X_{k}\right\}_{k \in K_{a}}\right)}$ for any $\left(K_{a}\right)$ in the restricted sum. We have also used the obvious estimate 


$$
\exp \left[-\frac{1}{4} \sum_{k \in K_{a}} \mathscr{L}\left(X_{k}\right)-\frac{1}{4} \mathscr{L}\left(\left\{X_{k}\right\}_{k \in K_{a}}\right)\right] \leqq \exp \left[-\frac{1}{4} \mathscr{L}\left(X_{K_{a}}\right)\right],
$$

where $\mathscr{L}(X)$ denotes the length of the shortest tree on $X$ and possibly other points. But

$$
\begin{gathered}
\sum_{\left(K_{a}\right)}^{\prime} \frac{1}{A !} \sum_{\Gamma_{c}} \prod_{a} \exp \left[-\frac{1}{4} \mathscr{L}\left(X_{K_{a}}\right)-\frac{1}{4} \sum_{k \in K_{a}} \mathscr{L}\left(X_{k}\right)\right] \\
=\sum_{A=1}^{\infty} \frac{1}{A !} \sum_{\text {on }\{1, \ldots, A\}} \sum_{\left(K_{a}\right)} \prod_{a=1}^{A} \exp [\ldots],
\end{gathered}
$$

where now we restrict the sum over $\left(K_{a}\right)$ by demanding that for each line $\left(a, a^{\prime}\right)$ of the tree $\tau, X_{K_{a}} \cap X_{K_{a^{\prime}}} \neq \emptyset$. Let $\left(N_{j}\right), N_{j} \geqq 1, \sum N_{j}=N$ be the multiplicities of different sets $X_{k j} \equiv Y_{j}$ among $\left(X_{k}\right), k=1, \ldots, N$. For $K \subset\{1, \ldots, N\}$, let $\underline{K}=\left(n_{\underline{K} j}\right), n_{\underline{k} j} \geqq 0$, be the multiplicities of $Y_{j}$ among $\left(X_{k}\right), k \in K$. Notice that the sequence $\left(n_{\underline{\underline{K}} j}\right)$ determines $X_{K}$, which we shall also denote as $X_{\underline{K}}$. It is convenient to replace the sum over $\left(K_{a}\right)_{a=1}^{A}$ in (A.48) by the sum over $\left(\underline{K}_{a}^{-}\right)_{a=1}^{A}$ with the same constraints:

$$
\begin{aligned}
(\mathrm{A} .48)= & \prod_{j} N_{j} ! \sum_{A=1}^{\infty} \frac{1}{A !} \sum_{\tau} \sum_{\left.\sum_{\underline{\underline{K}}}\right)} \prod_{a}\left(\prod_{j} n_{\underline{\underline{\underline{K}}} a j} !\right)^{-1} \\
& \cdot \exp \left[-\frac{1}{4} \mathscr{L}\left(X_{\underline{\underline{K}} a}\right)-\frac{1}{4} \sum_{j} n_{\underline{\underline{K}} a j} \mathscr{L}\left(Y_{j}\right)\right] .
\end{aligned}
$$

We shall need the following estimate

$$
\sum_{\underline{K}: X_{\underline{K}} \cap Y \neq \emptyset,\left|X_{K}\right|=S} e^{-\frac{1}{4} \mathscr{L}\left(X_{\underline{K}}\right)} \prod_{j} \exp \left[-n_{\underline{K} j}\left(C\left|Y_{j}\right|+\frac{1}{4} \mathscr{L}\left(Y_{j}\right)\right)\right] \leqq|Y| \varepsilon^{S},
$$

for some $\varepsilon\left(<\frac{1}{e}\right)$ and $C$ big enough.

The sum in (A.50) is estimated by the method of combinatorial coefficients [16]: $\sum a_{i} \leqq \max a_{i} b_{i}$, if $\sum b_{i}^{-1} \leqq 1, a_{i}, b_{i} \geqq 0$.

We can choose $K$ with the required properties in the following steps:

I. Choose $X_{\underline{\underline{K}}}$ with $X_{\underline{\underline{K}}} \cap Y \neq \emptyset,\left|X_{\underline{\underline{K}}}\right|=S$.

II. Choose a set $Q \subset X_{\underline{K}}$.

III. Choose a number $n>0$.

IV. For each $x \in Q$, choose $n_{x}>0$ so that $\sum_{x \in Q} n_{x}=n$.

V. For each $x \in Q$, choose $n_{x}$ times a set $X_{i} \ni x$.

The choices in which $X_{i}$ 's are sets $Y_{j}$ with multiplicities $\left(n_{j}\right), \sum n_{j}=n$ and $\cup X_{i}=X_{\underline{K}}$ give $\underline{K}$ with demanded properties. The sums over the choices I. to V. are controlled by the following combinatorial coefficients:

I.

II.

III.

IV.

V.

$$
|Y| C^{S} \exp \left(\frac{1}{4} \mathscr{L}\left(X_{\underline{K}}\right)\right)
$$


Altogether, by multiplying (A.51) to (A.55), we get a coefficient which for the choices leading to $K$ can be written as

$$
|Y| e^{\frac{1}{4} \mathscr{L}\left(X_{\underline{\underline{K}}}\right)} \prod_{j}\left(4^{n_{\underline{\underline{K}} J}} C^{n_{\underline{\underline{K}} J}\left|Y_{j}\right|} e^{\frac{1}{4} n_{\underline{\underline{K}}} \mathscr{L}\left(Y_{j}\right)}\right) .
$$

Inequality (A.50) follows.

Now notice that we may estimate the sum over $\left(K_{a}\right)$ in (A.49), given $\tau$, by successively using (A.50), starting with the outermost lines of $\tau$. This produces

$$
\begin{aligned}
(\mathrm{A} .49) \leqq & \prod_{j} N_{j} ! \prod_{k=1}^{N} C^{\left|I_{k}\right|} \sum_{A} \frac{1}{A !} \sum_{\tau} \sum_{(\underline{\underline{K}})}^{\prime \prime} \prod_{a} e^{-\frac{1}{4} \mathscr{L}\left(X_{\underline{\underline{K}}_{a}}\right)} \\
& \cdot \prod_{j} \exp \left[-n_{\underline{\underline{K}} a j}\left(C\left|Y_{j}\right|+\frac{1}{4} \mathscr{L}\left(Y_{j}\right)\right)\right] \\
\leqq & \prod_{j} ! \prod C^{\left|I_{k}\right|} \sum_{A} \frac{1}{A !} \sum_{\tau} \prod_{a} \sum_{S_{a}=1}^{\infty} \varepsilon^{+S_{a}} S_{a}^{d_{a}-1},
\end{aligned}
$$

where $d_{a}$ is the incidence number of the vertex $a$ of $\tau$. Using the estimate $\sum_{n \geqq 1} \varepsilon^{-n} n^{p} \leqq p ! \frac{\varepsilon}{1-\varepsilon}$ and the fact that there are $\frac{(A-2) !}{\prod\left(d_{a}-1\right) !}$ trees with the incidence numbers $d_{a}\left(\sum d_{a}=2 A-2\right)$ (cf. [15]), we obtain

$$
(\mathrm{A} .49) \leqq \prod_{j} N_{j} ! \prod_{k} C^{\left|I_{k}\right|} \sum_{A=1}^{\infty}\left(\frac{\varepsilon}{1-\varepsilon}\right)^{A} \leqq \prod N_{j} ! \prod C^{\left|I_{k}\right|}
$$

Equations (A.46), (A.48), (A.49), and (A.58) give finally

$$
\left|E^{T}\right| \leqq \prod_{k=1}^{N} C^{\left|I_{k}\right|} e^{\frac{1}{2} \mathscr{L}\left(X_{k}\right)}\left(\prod_{\Delta}\left|I_{k \Delta}\right| !\left|J_{k \Delta}\right| !\right)^{-\alpha} \prod_{j} N_{j} ! \exp \left[-\mathscr{L}\left(\left\{X_{k}\right\}_{k=1}^{N}\right)\right],
$$

from which (112) follows, since we could have put derivatives of fields in the expectation (A.32) as well.

Acknowledgements. We thank D. Brydges and G. Parisi for stressing the simplicity of fermions, and A.K. thanks L. Alvarez-Gaumé for helpful discussions. Both authors are grateful to the Physics Department of Harvard University and Professor A. Jaffe for hospitality, and K.G. also to Professor B. Simon and the Mathematics Department of Cal Tech.

\section{References}

1. Caianiello, E.: Number of Feynman graphs and convergence. Nuovo Cimento 3, 223-225 (1956)

2. 't Hooft, G.: Can we make sense out of "quantum chromodynamics". In: The whys of subnuclear physics, pp. 943-971. Zichichi, A. (ed.). New York, London: Plenum Press 1979, and Parisi, G.: The Borel transform and the renormalization group. Phys. Rep. 49, 215-219 (1979)

3. Mitter, P., Weisz, P.: Asymptotic scale invariance in a massive Thirring model with $U(n)$ symmetry. Phys. Rev. D 8, 4410-4429 (1973)

4. Gross, D., Neveu, A.: Dynamical symmetry breaking in asymptotically free field theories. Phys. Rev. D 10, 3235-3253 (1974)

5. Wilson, K., Kogut, J.: The renormalization group and the $\varepsilon$ expansion. Phys. Rep. 12 C, 75-200 (1974)

6. Wilson, K.: Model of coupling constant renormalization. Phys. Rev. D 2, 1438-1472 (1970) 
7. Gawędzki, K., Kupiainen, A., Tirozzi, B.: Renormalons: A dynamical system approach (to appear in Nucl. Phys. B)

8. Witten, E.: Chiral symmetry, the $\frac{1}{N}$ expansion and the $\mathrm{SU}(N)$ Thirring model. Nucl. Phys. B 145, 110-118 (1978)

9. Andrei, N., Lowenstein, J.: Diagonalization of the chiral invariant Gross-Neveu Hamiltonian. Phys. Rev. Lett. 43, 1698-1701 (1979)

10. Zamolodchikov, A., Zamolodchikov, A.: Factorized $S$-matrices in two dimensions as the exact solutions of certain relativistic quantum field theory models. Ann. Phys. 120, 253-291 (1979)

11. Gawędzki, K., Kupiainen, A.: Non-trivial continuum limit of a $\phi_{4}^{4}$ model with negative coupling constant. Cambridge (to appear in Nucl. Phys. B)

12. Iagolnitzer, D., Souillard, B.: On the analyticity in potential in classical statistical mechanics. Commun. Math. Phys. 60, 131-152 (1978)

Gawędzki, K., Kupiainen, A.: Renormalization group study of a critical lattice model. I. Commun. Math. Phys. 82, 407-433 (1981)

13. Thirring, W.: A soluble relativistic field theory. Ann. Phys. 3, 91-112 (1958)

14. Cammarota, C.: Decay of correlations for infinite range interactions in unbounded spin systems. Commun. Math. Phys. 85, 517-528 (1982)

15. Seiler, E.: Gauge theories as a problem of constructive quantum field theory and statistical mechanics. Lecture Notes in Physics, Vol. 159. Berlin, Heidelberg, New York: Springer 1982

16. Glimm, J., Jaffe, A.: Positivity of the $\phi_{3}^{4}$ Hamiltonian. Fortschr. Phys. 21, 327-376 (1973)

17. Feldman, J., Magnen, J., Rivasseau, V., Sénéor, R.: Massive Gross-Neveu model: a rigorous perturbative construction. Phys. Rev. Lett. 54, 1479-1481 (1985)

Communicated by A. Jaffe

Received March 29, 1985; in revised form June 7, 1985 